\title{
Synthesis of Pyridinylpyrrole Derivatives via the Palladium-Catalyzed Reaction of Acetylpyridines with Methyleneaziridines
}

Amal I. Siriwardana, Kalum K.A. D. S. Kathriarachchi, Itaru Nakamura, Ilya D. Gridnev, and Yoshinori Yamamoto*

Department of Chemistry, Graduate School of Science,

Tohoku University, Sendai 980-8578, Japan 


\section{Alternative Mechanism.}

One referee suggested the following mechanism. Alper has previously demonstrated ${ }^{1}$ that N-butylmethyleneaziridine is transformed into 1-n-butyl-3-methyleneazetidin-2-one in the presence of catalytic $\mathrm{Pd}(0)$ under an atmosphere of $\mathrm{CO}$ via $\mathrm{Pd}$-mediated insertion of $\mathrm{CO}$ into the $\mathrm{sp}^{2} \mathrm{C}-\mathrm{N}$ bond. This transformation likely occurs via oxidative addition of the methyleneaziridine to $\operatorname{Pd}(0)$ to afford an exo-methylene azametallacyclobutane, which undergoes subsequent insertion of $\mathrm{CO}$ and reductive elimination to afford the product. The reaction described by the authors could therefore occur through a related mechanism involving initial oxidative addition of methyleneaziridine $\mathrm{sp}^{2} \mathrm{C}-\mathrm{N}$ bond to $\operatorname{Pd}(0)$ [rather than $\mathrm{C}-\mathrm{H}$ activation]. This would provide and azametallacyclobutane that could react with the acetophenone, undergoing a proton transfer/sigma bond metathesis between the $\mathrm{C}-\mathrm{H}$ bond and the $\mathrm{Pd}-\mathrm{N}$ bond to afford a vinylpalladium enolate species. Reductive elimination would lead to a 1-vinylacetophenone bearing a methyleneamino substituent. Intramolecular condensation of the nitrogen with the carbonyl followed by tautomerization would provide the observed product.

Accordingly, we carried out the NMR experiments. We have checked the reactions of acetophenone 9 and benzylaziridine 3a with $\mathrm{Pd}\left(\mathrm{PPh}_{3}\right)_{4}$ in deuteriotoluene in an NMR tube separately. We have failed to observe any interaction between 3a and palladium 
even after prolonged heating at $110{ }^{\circ} \mathrm{C}$. On the other hand, after overnight heating of the deuteriotoluene solution containing an equivalent amount of $\mathbf{9}$ and palladium, formation of some new product has been detected (approximately $1 \%$ yield). The reaction was accompanied with the decomposition of palladium yielding Pd mirror on the NMR tube. The ${ }^{1} \mathrm{H}$ and ${ }^{13} \mathrm{C}$ NMR spectra of this new product resembled closely those of 9 (the signal in the aliphatic region of the NMR spectra belonged to a $\mathrm{CH}_{3}$ group as shown by DEPT experiments), but its extremely low concentration precluded the accurate structure elucidation. Thus, the NMR experiments suggested that 9 is more reactive towards the catalyst compared to $\mathbf{3 a}$ that is consistent with the $\mathrm{C}-\mathrm{H}$ activation mechanism of the whole transformation. Furthermore, as mentioned later in eq 3 of the text, we have obtained a strong support for the hydropalladation mechanism.

\section{$d$-Content of the recovered 9- $d_{3}$}

A reviewer pointed that some loss of deuterium would be observed in the recovered deuterated acetophenone. We investigated the d-content at methyl position of the recovered $9-d_{3}$, and found that it was $55 \%$. This result supports that the equilibrium between $\mathbf{9 - d _ { 3 }}$ and $\mathbf{1 3}$ shown in eq 3 exists really in the addition reaction. 
General. Spectroscopic measurements were carried out with the following instruments: JEOL JNM AL-400 and JEOL $\alpha-500\left({ }^{1} \mathrm{H}\right.$ NMR, ${ }^{13} \mathrm{C}$ NMR), SHIMADZU FTIR-8200A (FT-IR), HITACHI M-2500s (HRMS). All Chemicals were purchased from Aldrich Chemical Company. All methyleneaziridines were prepared following the reported procedure. $^{2}$

The Preparation of $1 \mathrm{~d}$ as a representative procedure for the synthesis of pyridinylpyrroles. To the mixture of $\mathrm{Pd}\left(\mathrm{PPh}_{3}\right)_{4}(104.0 \mathrm{mg}, 0.09 \mathrm{mmol})$ and acetylpyriidne 4a $(67 \mu \mathrm{l}, 0.6 \mathrm{mmol})$ was added methyleneaziridine $\mathbf{3 d}$ (49.5 mg, 0.3 mmol) under Ar atmosphere in a pressure vial and the mixture was stirred at $120{ }^{\circ} \mathrm{C}$ for 3 days. After completion of the reaction, which was monitored by GC, the mixture was filtered through a short Florisil column with ethyl acetate as eluent. Purification of the crude product with Florisil column chromatography (hexane : ethylacetate $=20: 1$ ) afforded the pyridinylpyrrole $\mathbf{1 d}$ in $87 \%$ yield.

\section{General experimental procedure for the preparation of 10 as an authentic}

sample. $^{3 \mathrm{a}}$ To a solution of LDA, prepared from diisopropylamine $(0.588 \mathrm{~g}, 5 \mathrm{mmol})$ and $n$-butyllithium (1.6 M hexane solution, $3.1 \mathrm{ml}, 5 \mathrm{mmol}$ ) in THF was added $\mathrm{TMSCHN}_{2}{ }^{3 \mathrm{~b}}$ (1.56 $\mathrm{M}$ hexane solution, $3.2 \mathrm{ml}, 5 \mathrm{mmol})$ at $-78{ }^{\circ} \mathrm{C}$ under an $\mathrm{Ar}$ atmosphere. After 
stirring for $30 \mathrm{~min}$, a solution of 1-(dibenzylamino)-2-propanone ${ }^{3 \mathrm{c}}(1.013 \mathrm{~g}, 4 \mathrm{mmol})$, prepared from 1-chloro-2-propanone $(0.930 \mathrm{~g}, 10 \mathrm{mmol})$, dibenzylamine $(0.977 \mathrm{~g}, 5$ $\mathrm{mmol})$, and $\mathrm{Et}_{3} \mathrm{~N}(0.611 \mathrm{~g}, 6 \mathrm{mmol})$ in $\mathrm{THF}$, was added and the whole mixture was stirred at $-78{ }^{\circ} \mathrm{C}$ for $3 \mathrm{~h}$, then heated under reflux for $2 \mathrm{~h}$. The reaction mixture was cooled to ambient temperature and $50 \mathrm{ml}$ of cold water was added. The mixture was filtered off and the filtrate was extracted with benzene twice. The combined organic layer was dried over magnesium sulphate. The solvent was evaporated and the residue was used without further purification in the next step. The mixture of residue and manganese dioxide $(3.476 \mathrm{~g}, 40 \mathrm{mmol})$ in dichloromethane $(40 \mathrm{ml})$ was heated under reflux for $3 \mathrm{~h}$ to give $\mathbf{1 0 .}$

Reference

(1) Hamel, N.; Alper, H. Tetrahedron Lett. 1987, 28, 3237.

(2) Preparation of methyleneaziridines: (a) De Kimpe, N.; De Smaele, D.; Sakonyi, Z. J. Org. Chem. 1997, 62, 2448. (b) Ince, J.; Ross, T. M.; Slawin, A. M. Z.; Ennis, D. S. Tetrahedron 1996, 52, 7037.

(3) For authentic sample preparation, (a) Ogawa, H.; Aoyama, T.; Shioiri, T. Heterocycles 1996, 42, 75. (b) Miwa, K.; Aoyama, T.; Shioiri, T. Synlett 1994, 109. (c) Stratford, E.; Curley, R. W. J. Med. Chem. 1983, 26, 1463. 
2-Pyridinyl-(1-Benzyl-4-methyl)pyrrole (1a). IR (neat) 3384, 3063, 3030, 2925, 2868, 1947, 1589, 1560, 1501, 1477, 1402, 1348, 1284, 1259, 1216, 1178, 1152, 1136, 1090, 1076, 1027, 1001, 985, 777, $735 \mathrm{~cm}^{-1} ;{ }^{1} \mathrm{H}$ NMR $\left(\mathrm{CDCl}_{3}, 500 \mathrm{MHz}\right) \delta 2.11(\mathrm{~s}, 3 \mathrm{H}), 5.68$ (s, 2H), $6.44(\mathrm{~s}, 1 \mathrm{H}), 6.55(\mathrm{~s}, 1 \mathrm{H}), 6.99(\mathrm{t}, J=6.2 \mathrm{~Hz}, 1 \mathrm{H}), 7.00(\mathrm{~d}, J=7.0 \mathrm{~Hz}, 2 \mathrm{H})$, 7.16-7.24 (m, 3H), 7.42-7.46 (m, 1H), 7.54-7.60 (m, 1H), $8.47(\mathrm{~d}, J=4.5 \mathrm{~Hz}, 1 \mathrm{H}) ;{ }^{13} \mathrm{C}$ NMR $\left(\mathrm{CDCl}_{3}, 125 \mathrm{MHz}\right) \delta 11.80,51.54,112.42,118.87,120.09,121.27,124.07$, 126.89, 127.14, 127.22, 128.33, 128.72, 131.73, 136.18, 139.67, 148.44, 152.55; Anal. Calcd for $\mathrm{C}_{17} \mathrm{H}_{16} \mathrm{~N}_{2}$ (248.32): C, 82.22; H, 6.49; N, 11.28. Found: C, 82.08; H, 6.51; N, 11.10. HRMS (EI) Calcd for $\mathrm{C}_{17} \mathrm{H}_{16} \mathrm{~N}_{2}: \mathrm{m} / \mathrm{z} 248.3224$. Found: $\mathrm{m} / \mathrm{z} 248.1308$.

2-Pyridinyl-[4-Methyl-1-(1-phenyl-ethyl)]pyrrole (1b). IR (neat) 3864, 3842, 3817, $3748,3689,3670,3633,2918,2848,2355,1719,1696,1653,1590,1501,1437,1390$, 1187, 1152, 1090, 1019, 778, $738 \mathrm{~cm}^{-1} ;{ }^{1} \mathrm{H} \mathrm{NMR}\left(\mathrm{CDCl}_{3}, 400 \mathrm{MHz}\right) \delta 1.79(\mathrm{~d}, J=6.8 \mathrm{~Hz}$, 3H), $2.10(\mathrm{~s}, 3 \mathrm{H}), 6.38(\mathrm{~s}, 1 \mathrm{H}), 6.66(\mathrm{~s}, 1 \mathrm{H}), 6.80(\mathrm{q}, J=7.2 \mathrm{~Hz}, 1 \mathrm{H}), 6.99-7.02(\mathrm{~m}, 1 \mathrm{H})$, 7.09-7.25 (m, 5H), $7.40(\mathrm{~d}, J=8.0 \mathrm{~Hz}, 1 \mathrm{H}), 7.53-7.57(\mathrm{~m}, 1 \mathrm{H}), 8.51(\mathrm{~d}, J=4.8 \mathrm{~Hz}, 1 \mathrm{H})$;

${ }^{13} \mathrm{C} \mathrm{NMR}\left(\mathrm{CDCl}_{3}, 100 \mathrm{MHz}\right) \delta 12.05,21.74,54.22,112.38,112.39,118.66,119.79$, $120.05,121.80,126.15,126.68,128.17,136.05,143.99,148.42$; Anal. Calcd for $\mathrm{C}_{18} \mathrm{H}_{18} \mathrm{~N}_{2}$ (262.35): C, 82.41; H, 6.92; N, 10.68. Found: C, 82.63; H, 7.09; N, 10.57. HRMS (EI) Calcd for $\mathrm{C}_{18} \mathrm{H}_{18} \mathrm{~N}_{2}: \mathrm{m} / \mathrm{z} 262.3490$. Found: $\mathrm{m} / \mathrm{z} 262.1465$.

2-Pyridinyl-(1-Hexyl-4-methyl)pyrrole (1c). IR (neat) 3876, 3845, 3828, 3810, 3774, $3696,3682,3660,3348,3070,2955,2925,2855,2361,1726,1589,1501,1452,1433$, 1312, 1261, 1201, 1153, 1119, 1089, 1027, 854, 778, 742, $720 \mathrm{~cm}^{-1} ;{ }^{1} \mathrm{H} \mathrm{NMR}\left(\mathrm{CDCl}_{3}\right.$, 
$400 \mathrm{MHz}) \delta 0.84(\mathrm{t}, J=7.1 \mathrm{~Hz}, 3 \mathrm{H}), 1.21-1.23(\mathrm{~m}, 6 \mathrm{H}), 1.65-1.67(\mathrm{~m}, 2 \mathrm{H}), 2.11(\mathrm{~s}, 3 \mathrm{H})$, $4.36(\mathrm{t}, J=8.4 \mathrm{~Hz}, 2 \mathrm{H}), 6.37(\mathrm{~s}, 1 \mathrm{H}), 6.55(\mathrm{~s}, 1 \mathrm{H}), 7.00-7.03(\mathrm{~m}, 1 \mathrm{H}), 7.46(\mathrm{~d}, J=6.2 \mathrm{~Hz}$, $1 \mathrm{H}), 7.57-7.61(\mathrm{~m}, 1 \mathrm{H}), 8.51(\mathrm{~d}, J=4.4 \mathrm{~Hz}, 1 \mathrm{H}) .{ }^{13} \mathrm{C} \mathrm{NMR}\left(\mathrm{CDCl}_{3}, 100 \mathrm{MHz}\right) \delta 11.83$, $14.07,22.57,26.42,31.40,31.82,48.52,111.91,117.96,119.83,121.17,123.55,131.17$, 135.94, 148.43, 152.76; Anal. Calcd for $\mathrm{C}_{16} \mathrm{H}_{22} \mathrm{~N}_{2}$ (242.36): C, 79.29; H, 9.15; N, 11.56.

Found: C, 79.14; H, 9.44; N, 11.37. HRMS (EI) Calcd for $\mathrm{C}_{16} \mathrm{H}_{22} \mathrm{~N}_{2}: \mathrm{m} / \mathrm{z} 242.3594$. Found: m/z 242.1778.

2-Pyridinyl-[1-(1-Cyclohexyl-ethyl)-4-methyl]pyrrole (1d). IR (neat) 2956, 2925, $2855,1621,1538,1506,1455,1375,1322,1272,1219,1145,1118,1031,898,860,789$, $754 \mathrm{~cm}^{-1} ;{ }^{1} \mathrm{H}$ NMR $\left(\mathrm{CDCl}_{3}, 400 \mathrm{MHz}\right) \delta 0.63-0.73(\mathrm{~m}, 1 \mathrm{H}), 0.84-0.88(\mathrm{~m}, 1 \mathrm{H}), 0.90-1.07$ (m, 2H), 1.15-1.20 (m, 2H), 1.44-1.54 (m, 6H), 1.67-1.75 (m, 2H), $2.12(\mathrm{~s}, 3 \mathrm{H}), 4.97$ (q, $J=6.8 \mathrm{~Hz}, 1 \mathrm{H}), 6.25(\mathrm{~s}, 1 \mathrm{H}), 6.65((\mathrm{~s}, 1 \mathrm{H}), 7.03(\mathrm{t}, J=7.2 \mathrm{~Hz}, 1 \mathrm{H}), 7.42(\mathrm{~d}, J=7.9 \mathrm{~Hz}$, 1H), $7.60(\mathrm{~d}, J=7.6 \mathrm{~Hz}, 1 \mathrm{H}), 8.53(\mathrm{~d}, J=4.9 \mathrm{~Hz}, 1 \mathrm{H}) ;{ }^{13} \mathrm{C} \mathrm{NMR}\left(\mathrm{CDCl}_{3}, 100 \mathrm{MHz}\right) \delta$ $12.09,19.25,26.22,26.35,29.60,29.94,45.50,56.13,111.13,111.15,118.46,118.63$, 119.92, 122.20, 135.95, 148.55; Anal. Calcd for $\mathrm{C}_{18} \mathrm{H}_{24} \mathrm{~N}_{2}$ (268.40): C, 80.55; H, 9.01 N, 10.44 Found: C, 80.32; H, 9.29; N, 10.39. HRMS (EI) Calcd for $\mathrm{C}_{18} \mathrm{H}_{24} \mathrm{~N}_{2}: \mathrm{m} / \mathrm{z} 268.3966$. Found: m/z 268.2934. 
2-Pyridinyl-\{1-[1-(4-Chloro-phenyl)-ethyl]-4-methyl\}pyrrole (1e). IR (neat) 3388, $3047,2925,2856,1709,1589,1561,1502,1447,1408,1350,1284,1259,1177,1153$, 1135, 1091, 1015, 805, $778 \mathrm{~cm}^{-1} ;{ }^{1} \mathrm{H}$ NMR $\left(\mathrm{CDCl}_{3}, 400 \mathrm{MHz}\right) \delta 2.04(\mathrm{~s}, 3 \mathrm{H}), 5.59(\mathrm{~s}$, 2H), $6.46(\mathrm{~s}, 1 \mathrm{H}), 6.47(\mathrm{~s}, 1 \mathrm{H}), 6.92(\mathrm{~d}, J=7.8 \mathrm{~Hz}, 3 \mathrm{H}), 7.11(\mathrm{~d}, J=6.4 \mathrm{~Hz}, 2 \mathrm{H}), 7.38(\mathrm{~d}$, $J=7.9 \mathrm{~Hz}, 1 \mathrm{H}), 7.47-7.52(\mathrm{~m}, 1 \mathrm{H}), 8.38(\mathrm{~d}, J=4.6 \mathrm{~Hz}, 1 \mathrm{H}), ;{ }^{13} \mathrm{C} \mathrm{NMR}\left(\mathrm{CDCl}_{3}, 100\right.$ MHz) $\delta 11.86,51.11,112.47,119.02,120.09,121.06,123.94,128.20,128.39,131.55$, 132.53, 136.07, 138.24, 148.36, 152.38; Anal. Calcd for $\mathrm{C}_{18} \mathrm{H}_{15} \mathrm{ClN}_{2}$ (282.77): C, 72.21; H, 5.35; Cl, 12.54; N, 9.91. Found: C, 72.49; H, 5.39; N, 9.75. HRMS (EI) Calcd for $\mathrm{C}_{17} \mathrm{H}_{15} \mathrm{ClN}_{2}: \mathrm{m} / \mathrm{z} 282.7672$. Found: m/z 282.7918.

2-Pyridinyl-[1-(2,2-Dimethoxy-ethyl)-4-methyl]pyrrole (1f). IR (neat) 3348, 2930, 2834, 2360, 1707, 1590, 1560, 1502, 1447, 1387, 1316, 1284, 1262, 1193, 1125, 1076, 1027, 980, 921, 842, 779, $741 \mathrm{~cm}^{-1} ;{ }^{1} \mathrm{H}$ NMR $\left(\mathrm{CDCl}_{3}, 300 \mathrm{MHz}\right) \delta 2.11(\mathrm{~s}, 3 \mathrm{H}), 3.35$ (s, $6 \mathrm{H}), 4.46(\mathrm{~d}, J=5.3 \mathrm{~Hz}, 2 \mathrm{H}), 4.66(\mathrm{t}, J=5.1 \mathrm{~Hz}, 1 \mathrm{H}), 6.42(\mathrm{~s}, 1 \mathrm{H}), 6.61(\mathrm{~s}, 1 \mathrm{H}), 7.03(\mathrm{t}, J$ $=4.2 \mathrm{~Hz}, 1 \mathrm{H}), 7.50(\mathrm{~d}, J=7.5 \mathrm{~Hz}, 1 \mathrm{H}), 7.60(\mathrm{t}, J=7.3 \mathrm{~Hz}, 1 \mathrm{H}), 8.49(\mathrm{~d}, J=4.9 \mathrm{~Hz}, 1 \mathrm{H})$;

${ }^{13} \mathrm{C} \mathrm{NMR}\left(\mathrm{CDCl}_{3}, 75 \mathrm{MHz}\right) \delta 11.72,50.73,55.06,105.02,112.27,118.23,119.93$, 121.06, 125.32, 136.19, 148.26, 152.58; Anal. Calcd for $\mathrm{C}_{14} \mathrm{H}_{18} \mathrm{~N}_{2} \mathrm{O}_{2}$ (246.30): C, 68.27; 
H, 7.37; N, 11.37; O, 12.99. Found: C, 67.98; H, 7.53; N, 11.07. HRMS (EI) Calcd for $\mathrm{C}_{14} \mathrm{H}_{18} \mathrm{~N}_{2} \mathrm{O}_{2}: \mathrm{m} / \mathrm{z} 246.3050$. Found: $\mathrm{m} / \mathrm{z} 246.1363$.

2-Pyridinyl[1-(3-Methoxy-propyl)-4-methyl]pyrrole (1g). IR (neat) 3336, 2925, 2872, 1699, 1588, 1572, 1501, 1437, 1407, 1290, 1200, 1117, 996, 904, 788, $759 \mathrm{~cm}^{-1} ;{ }^{1} \mathrm{H}$ NMR $\left(\mathrm{CDCl}_{3}, 400 \mathrm{MHz}\right) \delta 1.92-1.98(\mathrm{~m}, 2 \mathrm{H}), 2.11(\mathrm{~s}, 3 \mathrm{H}), 3.27-3.30(\mathrm{~m}, 5 \mathrm{H}), 4.47(\mathrm{t}, J$ $=7.2 \mathrm{~Hz}, 2 \mathrm{H}), 6.40(\mathrm{~s}, 1 \mathrm{H}), 6.55(\mathrm{~s}, 1 \mathrm{H}), 7.01(\mathrm{t}, J=4.3 \mathrm{~Hz}, 1 \mathrm{H}), 7.47(\mathrm{~d}, J=7.1 \mathrm{~Hz}, 1 \mathrm{H})$, $7.60(\mathrm{t}, J=7.2 \mathrm{~Hz}, 1 \mathrm{H}), 8.51(\mathrm{~d}, J=4.4 \mathrm{~Hz}, 1 \mathrm{H}) ;{ }^{13} \mathrm{C} \mathrm{NMR}\left(\mathrm{CDCl}_{3}, 100 \mathrm{MHz}\right) \delta 11.79$, $31.70,45.51,58.43,69.63,112.08,118.00,119.82,120.97,124.09,131.12,135.09$, 148.47, 152.65; Anal. Calcd for $\mathrm{C}_{14} \mathrm{H}_{18} \mathrm{~N}_{2}$ (230.31): C, 73.01; H, 7.88; N, 12.16; O, 6.95. Found: C, 73.15; H, 7.59; N, 12.18. HRMS (EI) Calcd for $\mathrm{C}_{14} \mathrm{H}_{18} \mathrm{~N}_{2} \mathrm{O}: \mathrm{m} / \mathrm{z} 230.3056$. Found: m/z 230.2414.

2-Pyridinyl[4-Methy-1-(1-phenyl-ethyl)]pyrrole (1h). IR (neat) 3864, 3842, 3817, $3748,3689,3670,3633,2918,2848,2354,1719,1696,1653,1590,1501,1437,1390$, $1187,1152,1090,1019,778,738 \mathrm{~cm}^{-1} ;{ }^{1} \mathrm{H}$ NMR $\left(\mathrm{CDCl}_{3}, 300 \mathrm{MHz}\right) \delta 1.78(\mathrm{~d}, J=9.5$ $\mathrm{Hz}, 3 \mathrm{H}), 2.15(\mathrm{~s}, 3 \mathrm{H}), 5.34(\mathrm{q}, J=9.2 \mathrm{~Hz}, 1 \mathrm{H}), 6.11(\mathrm{~s}, 1 \mathrm{H}), 6.71(\mathrm{~s}, 1 \mathrm{H}), 6.99$ (d, $J=9.0$ $\mathrm{Hz}, 2 \mathrm{H}), 7.20-7.30(\mathrm{~m}, 4 \mathrm{H}), 7.50(\mathrm{~d}, J=7.8 \mathrm{~Hz}, 1 \mathrm{H}), 8.47-8.53(\mathrm{~m}, 2 \mathrm{H}) ;{ }^{13} \mathrm{C} \mathrm{NMR}$ 
$\left(\mathrm{CDCl}_{3}, 100 \mathrm{MHz}\right) \delta 12.05,21.73,54.21,112.38,112.39,118.66,119.79,120.06,121.79$, 126.15, 126.68, 128.17, 136.05, 143.98, 148.46; Anal. Calcd for $\mathrm{C}_{18} \mathrm{H}_{18} \mathrm{~N}_{2}$ (262.35): C, 82.41; H, 6.92; N, 10.68. Found: C, 82.17; H, 7.05; N, 10.54. HRMS (EI) Calcd for $\mathrm{C}_{18} \mathrm{H}_{18} \mathrm{~N}_{2}: \mathrm{m} / \mathrm{z} 262.1465$. Found: $\mathrm{m} / \mathrm{z} 262.1464$.

2-Pyridinyl[Methyl-1-(1-phenyl-ethyl)]pyrrole (1i). IR (neat) 3086, 3061, 3027, 2978, 2928, 2871, 1951, 1878, 1735, 1697, 1586, 1564, 1496, 1450, 1421, 1385, 1352, 1296, $1241,1210,1181,1138,1105,1072,1047,1027,1000,969,937,913,877,845,794$, 743, $715 \mathrm{~cm}^{-1} ;{ }^{1} \mathrm{H}$ NMR $\left(\mathrm{CDCl}_{3}, 500 \mathrm{MHz}\right) \delta 1.79(\mathrm{~d}, J=7.0 \mathrm{~Hz}, 3 \mathrm{H}), 2.14(\mathrm{~s}, 3 \mathrm{H}), 5.49$ $(\mathrm{q}, J=7.0 \mathrm{~Hz}, 1 \mathrm{H}), 6.21(\mathrm{~s}, 1 \mathrm{H}), 6.73(\mathrm{~s}, 1 \mathrm{H}), 7.04(\mathrm{~d}, J=8.0 \mathrm{~Hz}, 2 \mathrm{H}), 7.15(\mathrm{~d}, J=4.5$ $\mathrm{Hz}, 2 \mathrm{H}), 7.22-7.31(\mathrm{~m}, 3 \mathrm{H}), 8.49(\mathrm{~d}, J=4.5 \mathrm{~Hz}, 2 \mathrm{H}) .{ }^{13} \mathrm{C} \mathrm{NMR}\left(\mathrm{CDCl}_{3}, 125 \mathrm{MHz}\right)$ $\delta 11.91,22.48,54.79,112.14,119.57,119.65,122.65,125.60,127.30,128.67,131.92$, 140.90, 143.24, 149.70; Anal. Calcd for $\mathrm{C}_{18} \mathrm{H}_{18} \mathrm{~N}_{2}$ (262.35): C, 82.09; H, 7.27; N, 10.64. Found: C, 81.93; H, 6.99; N, 10.39. HRMS (EI) Calcd for $\mathrm{C}_{18} \mathrm{H}_{18} \mathrm{~N}_{2}$ : m/z 262.3490. Found: m/z 262.2465.

2-etaneonyl-\{6-[4-Methyl-1-(1-phenyl-ethyl)\}pyrrole (1j). IR (neat) 3892, 3844, 3827, 3810, 3788, 3696, 3681, 3657, 3375, 2976, 2925, 2281, 1697, 1587, 1494, 1448, 1415, $1363,1295,1248,1188,1120,1040,1025,954,856,796,757 \mathrm{~cm}^{-1} ;{ }^{1} \mathrm{H}$ NMR $\left(\mathrm{CDCl}_{3}\right.$, 
$400 \mathrm{MHz}) \delta 1.80(\mathrm{~d}, J=7.2 \mathrm{~Hz}, 3 \mathrm{H}), 2.04(\mathrm{~s}, 3 \mathrm{H}), 2.48(\mathrm{~s}, 3 \mathrm{H}), 6.41(\mathrm{~s}, 1 \mathrm{H}), 6.56(\mathrm{~s}, 1 \mathrm{H})$, $6.82(\mathrm{q}, J=5.1 \mathrm{~Hz}, 1 \mathrm{H}), 7.07(\mathrm{~d}, J=4.2 \mathrm{~Hz}, 2 \mathrm{H}), 7.13-7.22(\mathrm{~m}, 3 \mathrm{H}), 7.57-7.71(\mathrm{~m}, 3 \mathrm{H})$;

${ }^{13} \mathrm{C} \mathrm{NMR}\left(\mathrm{CDCl}_{3}, 100 \mathrm{MHz}\right) \delta 12.00,14.20,21.64,22.72,26.03,54.41,113.06,117.95$, 119.11, 120.76, 125.09, 126.19, 126.94, 128.32, 131.01, 137.09, 152.14, 152.28; Anal. Calcd for $\mathrm{C}_{20} \mathrm{H}_{20} \mathrm{~N}_{2} \mathrm{O}$ (304.38): C, 78.92; H, 6.62; N, 9.20; O, 5.26. Found: C, 78.68; H, 6.87; N, 9.42. HRMS (EI) Calcd for $\mathrm{C}_{20} \mathrm{H}_{20} \mathrm{~N}_{2} \mathrm{O}: \mathrm{m} / \mathrm{z}$ 304.3854. Found: m/z 304.2570.

2-Pyrazinyl-[4-Methyl-1-(1-phenyl-ethyl)]pyrrole (1k). IR (neat) 3347, 3056, 2978, 2921, 1704, 1579, 1518, 1497, 1450, 1426, 1376, 1296, 1236, 1209, 1184, 1126, 1069 , $1016,973,844,785,762, \mathrm{~cm}^{-1} ;{ }^{1} \mathrm{H} \mathrm{NMR}\left(\mathrm{CDCl}_{3}, 400 \mathrm{MHz}\right) \delta 1.80(\mathrm{~d}, J=7.2 \mathrm{~Hz}, 3 \mathrm{H})$, $2.12(\mathrm{~s}, 3 \mathrm{H}), 6.53(\mathrm{~s}, 1 \mathrm{H}), 6.72-6.75(\mathrm{~m}, 2 \mathrm{H}), 7.10(\mathrm{~d}, J=6.8 \mathrm{~Hz}, 2 \mathrm{H}), 7.15-7.26(\mathrm{~m}, 3 \mathrm{H})$, $8.22(\mathrm{~s}, 1 \mathrm{H}), 8.39(\mathrm{~s}, 1 \mathrm{H}), 8.72(\mathrm{~s}, 1 \mathrm{H}) ;{ }^{13} \mathrm{C} \mathrm{NMR}\left(\mathrm{CDCl}_{3}, 100 \mathrm{MHz}\right) \delta 11.96,21.89$, $54.75,113.58,119.30,121.65,126.09,126.94,128.30,139.90,142.60,143.18,143.53$; Anal. Calcd for $\mathrm{C}_{17} \mathrm{H}_{17} \mathrm{~N}_{3}$ (263.34): C, 77.54; H, 6.51 N, 15.96. Found: C, 77.27; H, 6.75; $\mathrm{N}$, 15.92. HRMS (EI) Calcd for $\mathrm{C}_{17} \mathrm{H}_{17} \mathrm{~N}_{3}: \mathrm{m} / \mathrm{z}$ 263.3371. Found: $\mathrm{m} / \mathrm{z}$ 263.1417.

2-Arylyl-(4-methyl-2-benzyl)pyrrole (10). IR (neat) 3347, 3063, 3030, 2924, 2868, 1953, 1808, 1681, 1605, 1511, 1496, 1451, 1406, 1356, 1297, 1263, 1217, 1174, 1126, 
1074, 1029, 972, 881, 785, 762, $\mathrm{cm}^{-1} ;{ }^{1} \mathrm{H}$ NMR $\left(\mathrm{CDCl}_{3}, 400 \mathrm{MHz}\right) \delta 2.06(\mathrm{~s}, 3 \mathrm{H}), 4.99(\mathrm{~s}$, $2 \mathrm{H}), 6.03(\mathrm{~s}, 1 \mathrm{H}), 6.43(\mathrm{~s}, 1 \mathrm{H}), 7.16-7.24(\mathrm{~m}, 10 \mathrm{H}) ;{ }^{13} \mathrm{C} \mathrm{NMR}\left(\mathrm{CDCl}_{3}, 100 \mathrm{MHz}\right) \delta$ $11.98,50.47,110.18,120.86,126.46,126.71,127.15,128.25,128.54,128.64,133.29$, 138.95; Anal. Calcd for $\mathrm{C}_{18} \mathrm{H}_{17} \mathrm{~N}$ (247.33): C, 87.41; H, 6.93; N, 5.66. Found: C, 87.57; H, 6.78; N, 5.51. HRMS (E1) Calcd for $\mathrm{C}_{18} \mathrm{H}_{17} \mathrm{~N}: \mathrm{m} / \mathrm{z}$ 247.3343. Found: m/z 247.1356. 


\section{${ }^{1}$ H-NMR of 2-Pyridinyl-(1-Benzyl-4-methyl)pyrrole (1a).}

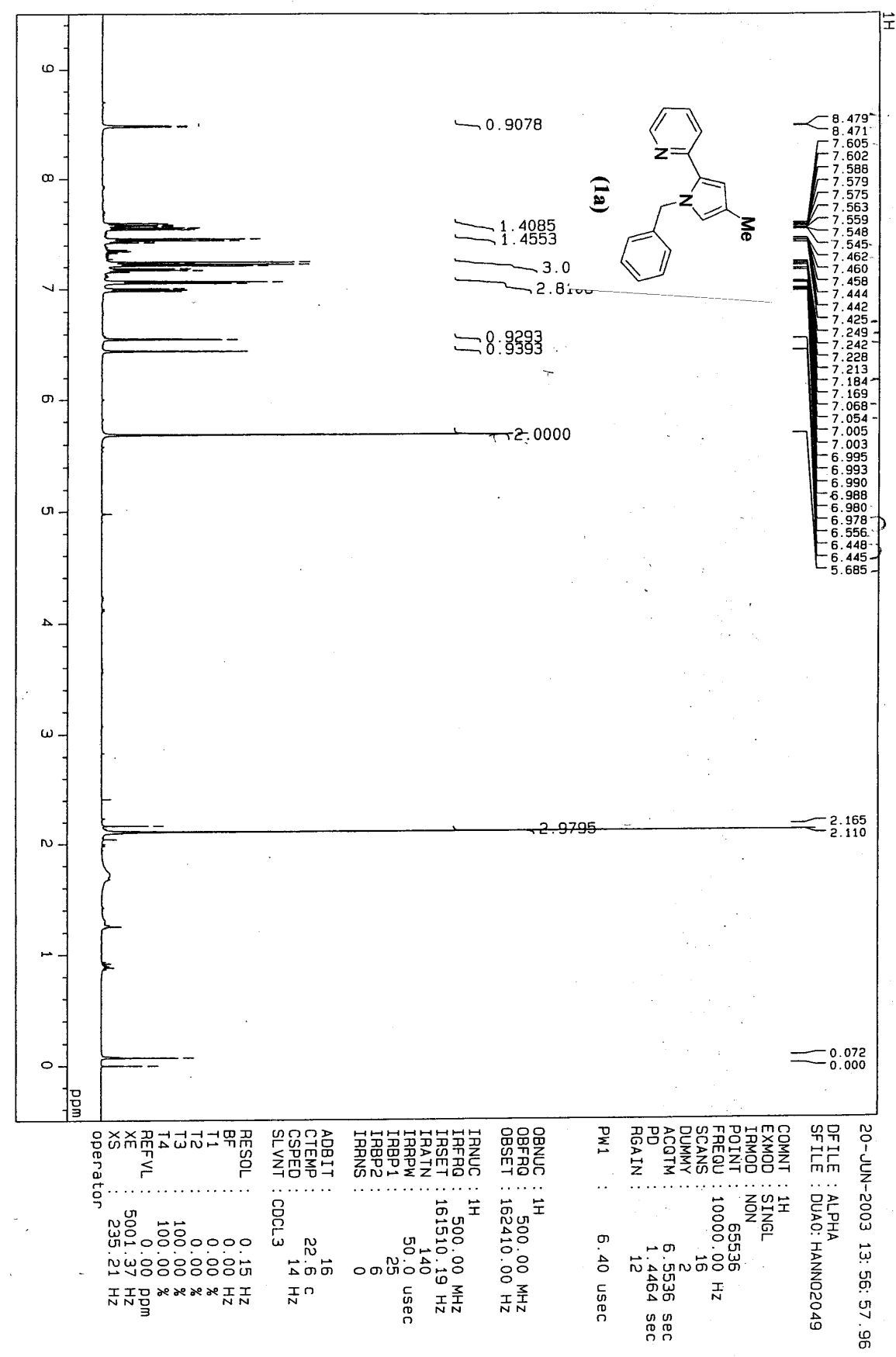




\section{${ }^{13}$ C-NMR of 2-Pyridinyl-(1-Benzyl-4-methyl)pyrrole (1a).}

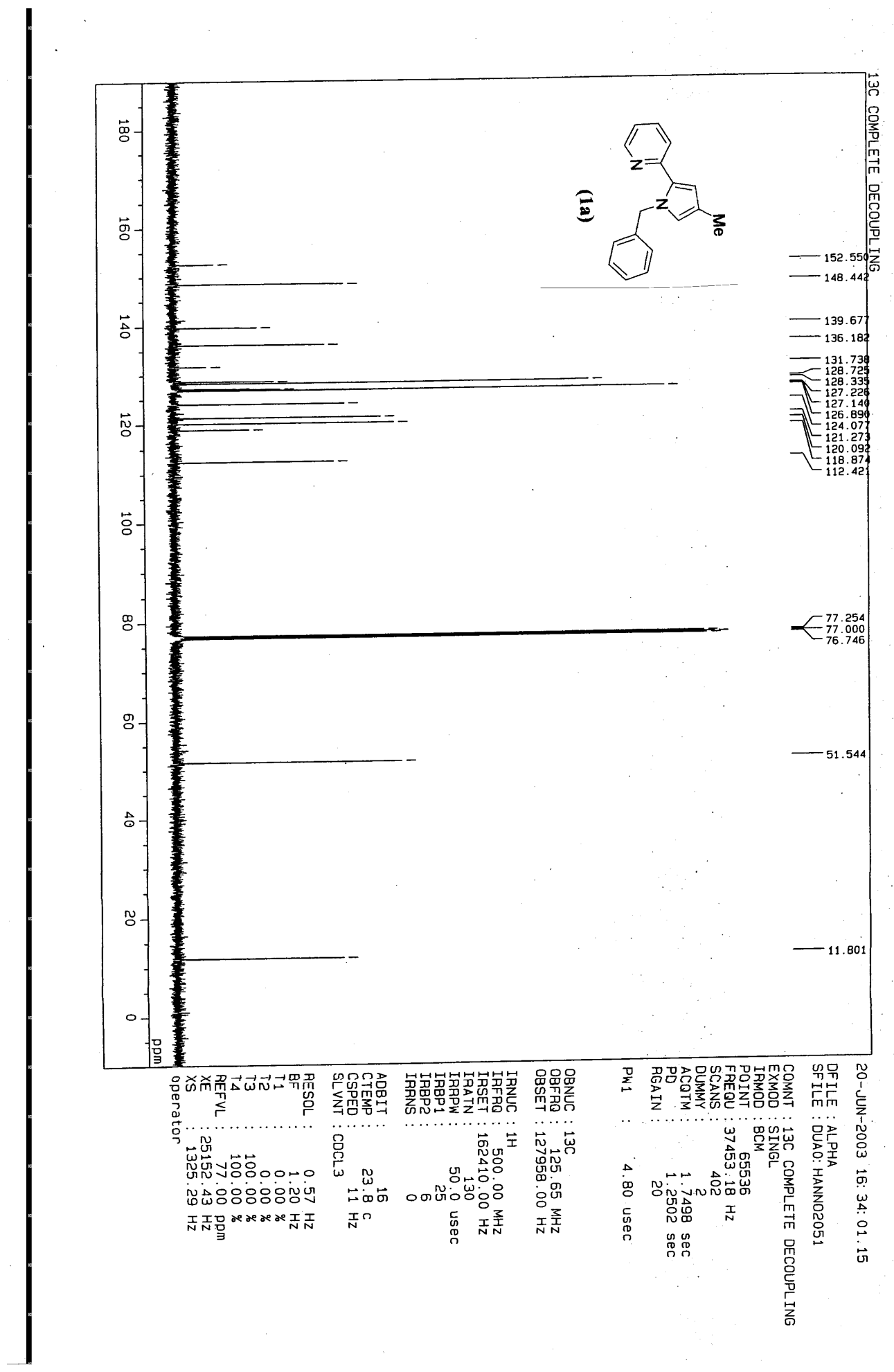




\section{${ }^{1}$ H-NMR of 2-Pyridinyl-[4-Methyl-1-(1-phenyl-ethyl)]pyrrole (1b).}

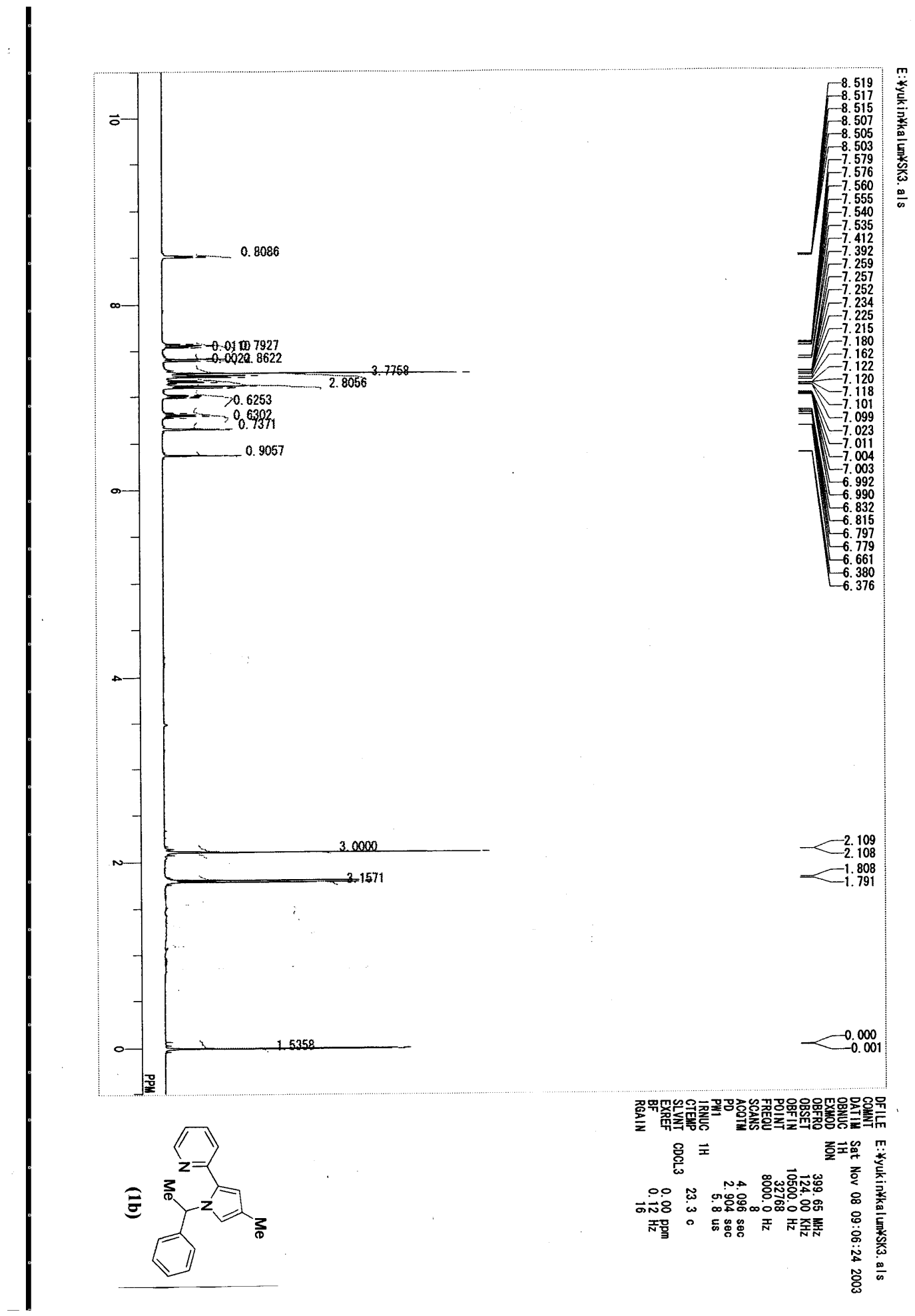


${ }^{13}$ C-NMR of 2-Pyridinyl-[4-Methyl-1-(1-phenyl-ethyl)]pyrrole (1b).

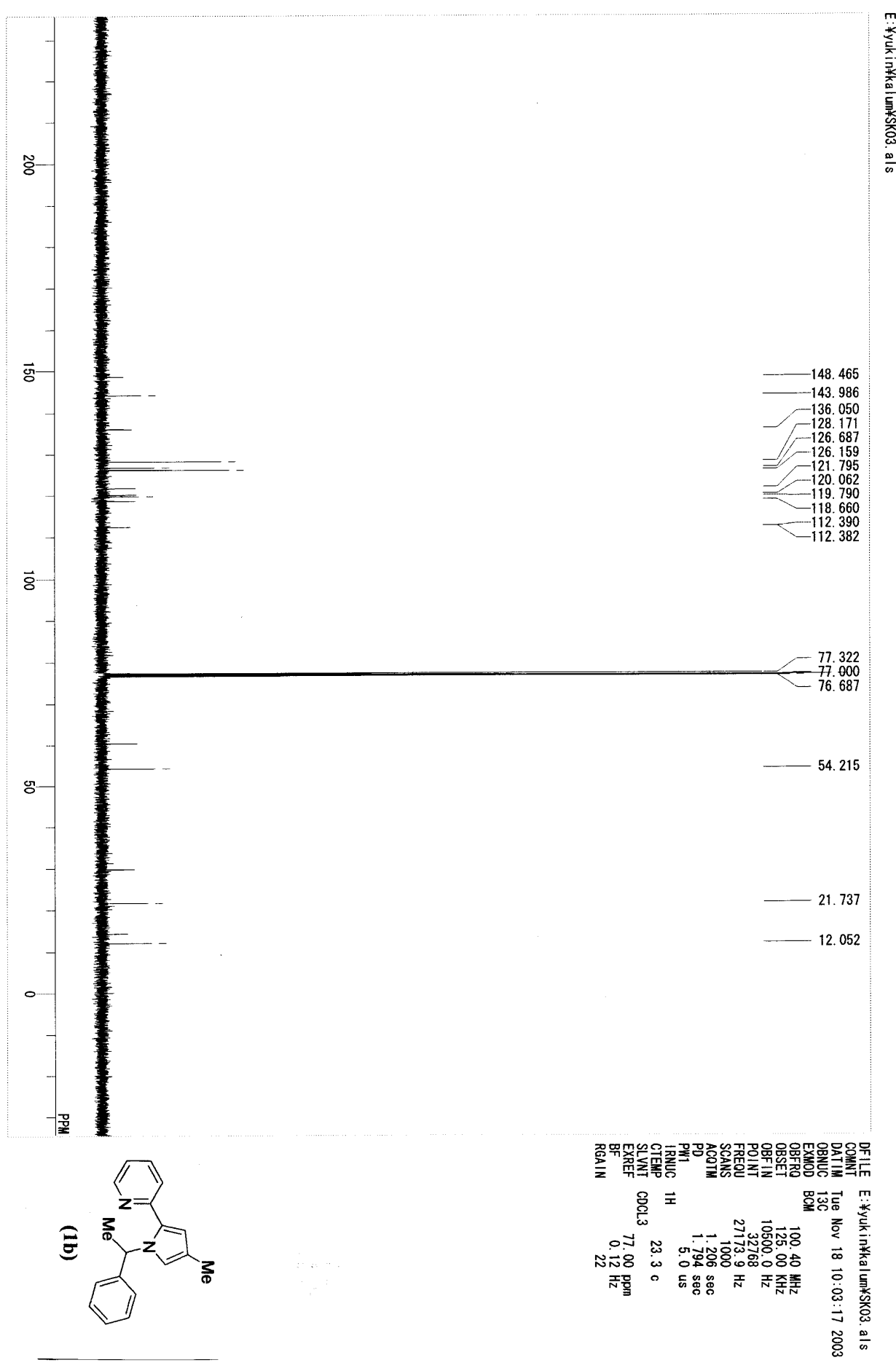


${ }^{1}$ H-NMR of 2-Pyridinyl-(1-Hexyl-4-methyl)pyrrole (1c).
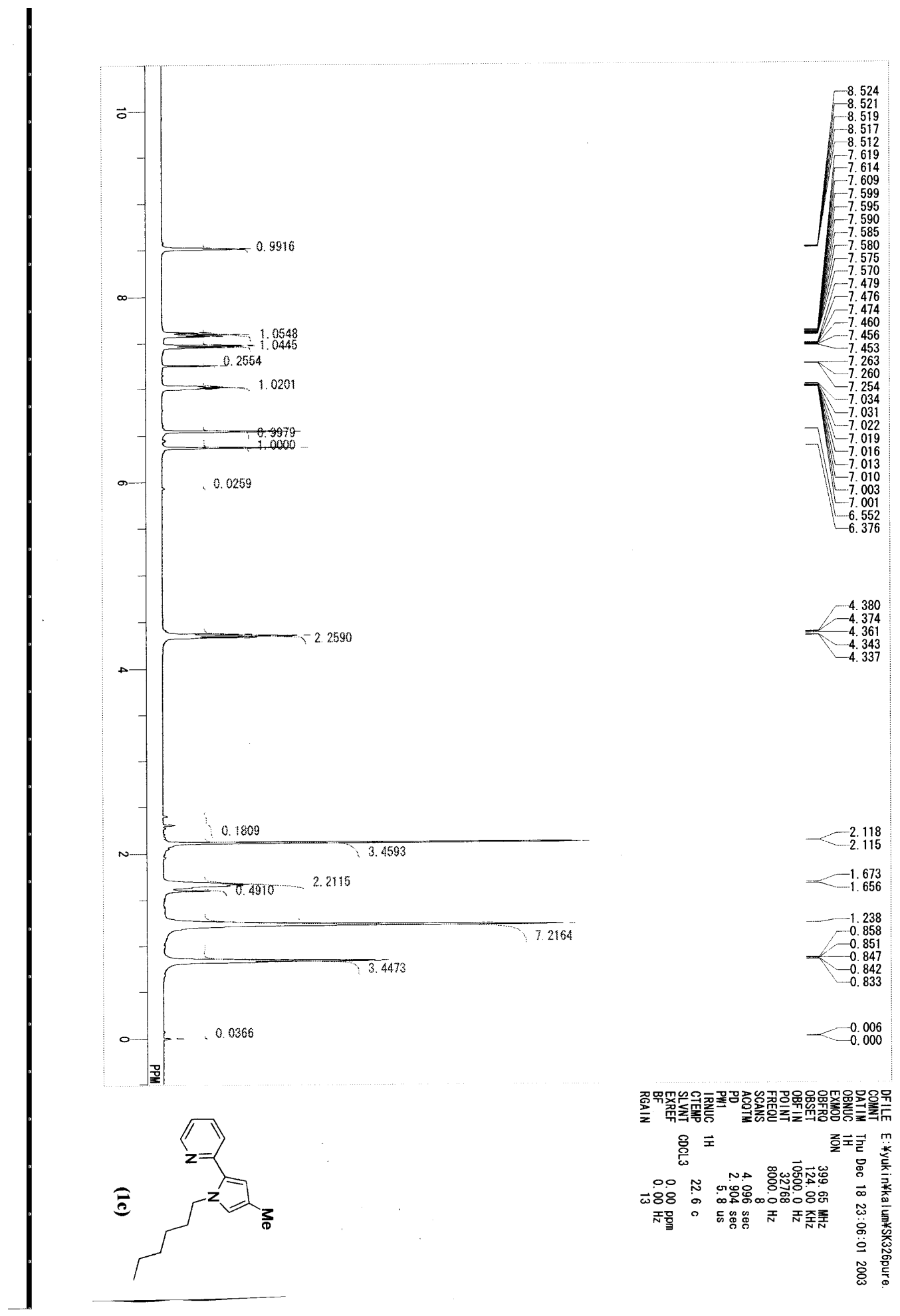

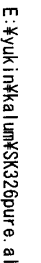

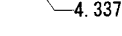

$\begin{array}{r}2.118 \\ -2.115 \\ \hline\end{array}$

1.673
-1.656
-1.238

$-1.238$

$-0.851$

$\begin{array}{r}-0.847 \\ -0.842 \\ \hline\end{array}$

$<-0.006$

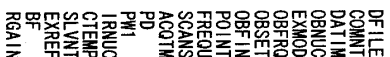

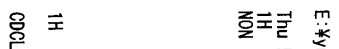

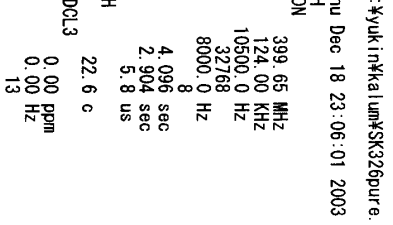




\section{${ }^{13}$ C-NMR of 2-Pyridinyl-(1-Hexyl-4-methyl)pyrrole (1c).}

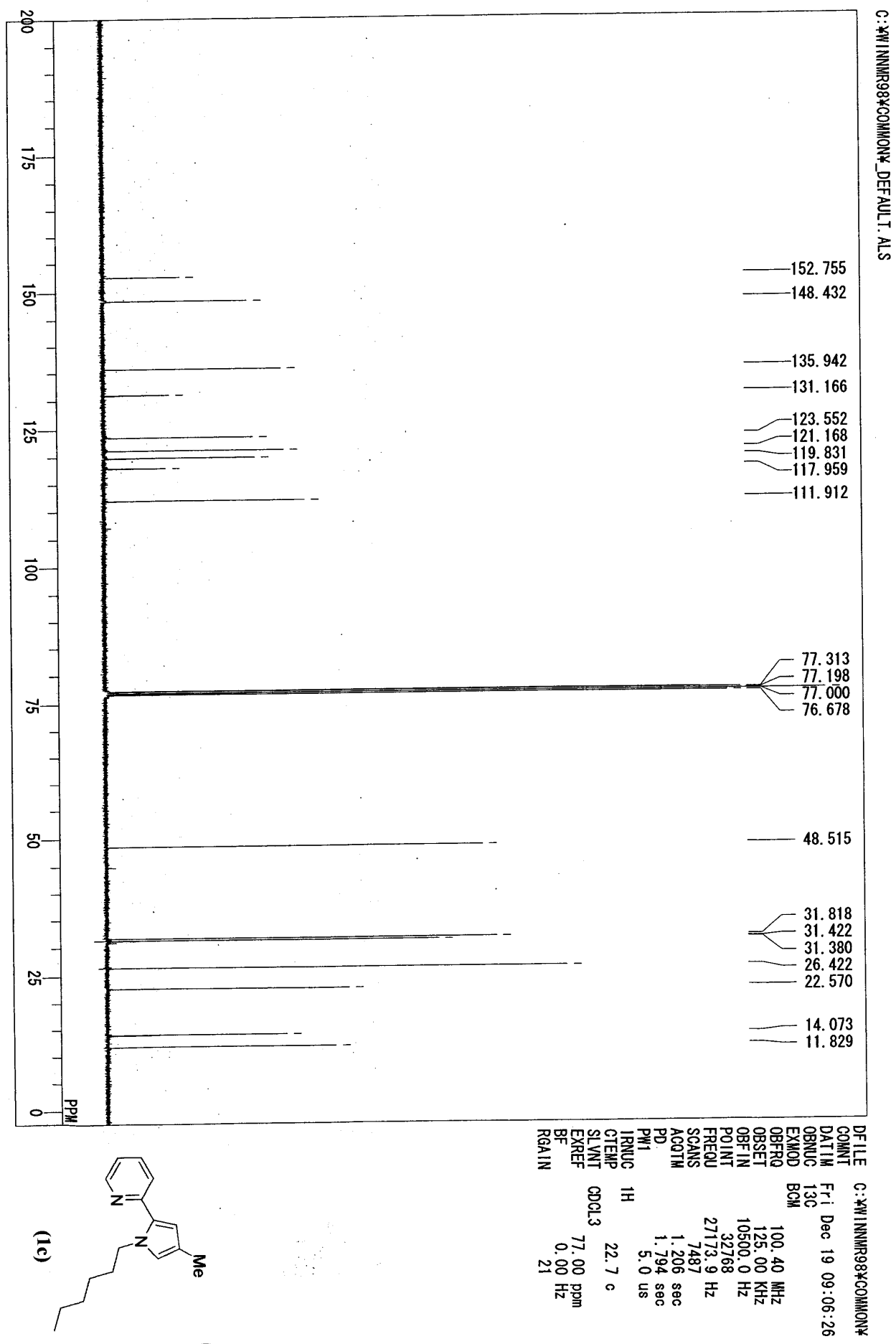




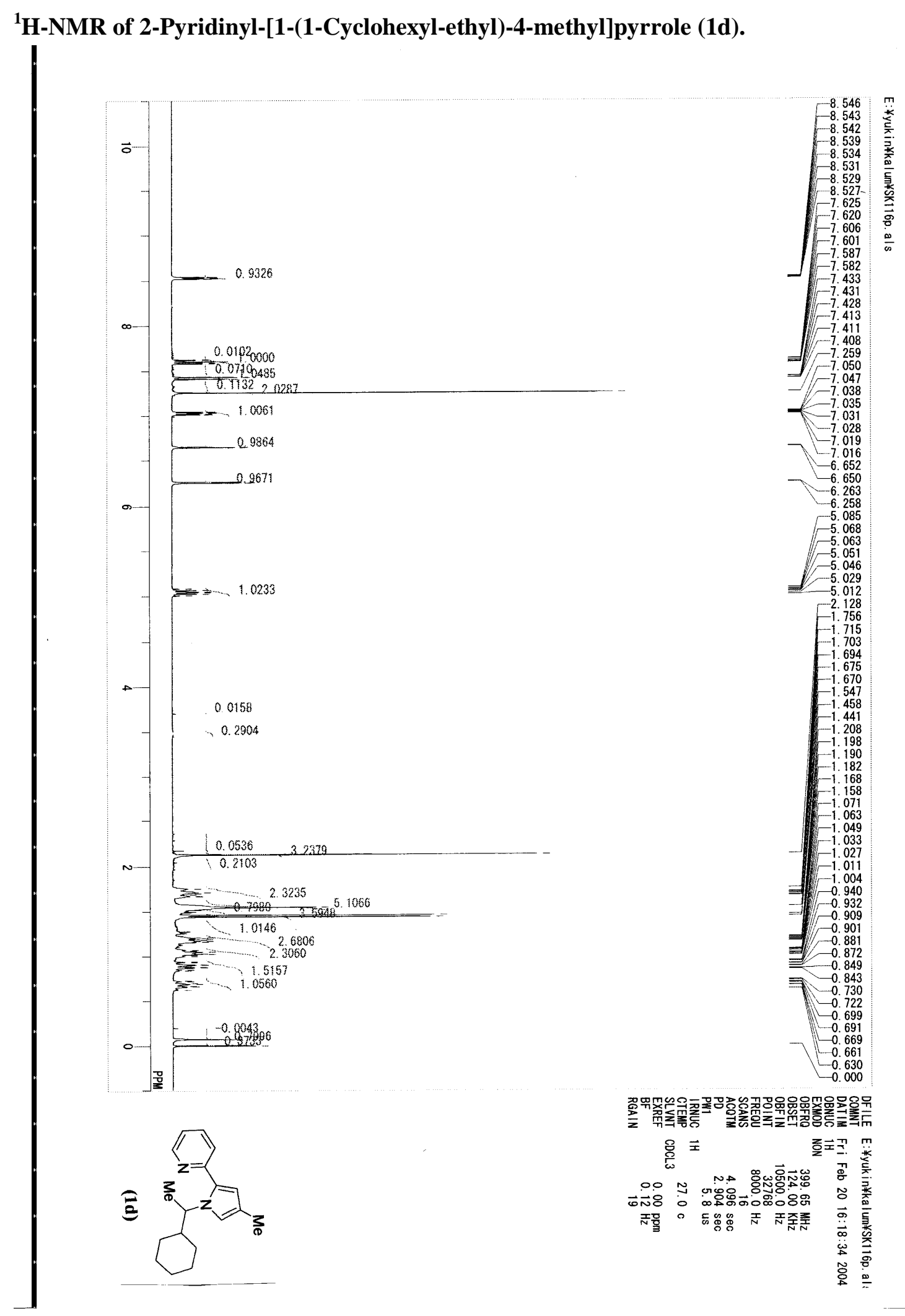


${ }^{13}$ C-NMR of 2-Pyridinyl-[1-(1-Cyclohexyl-ethyl)-4-methyl]pyrrole (1d).

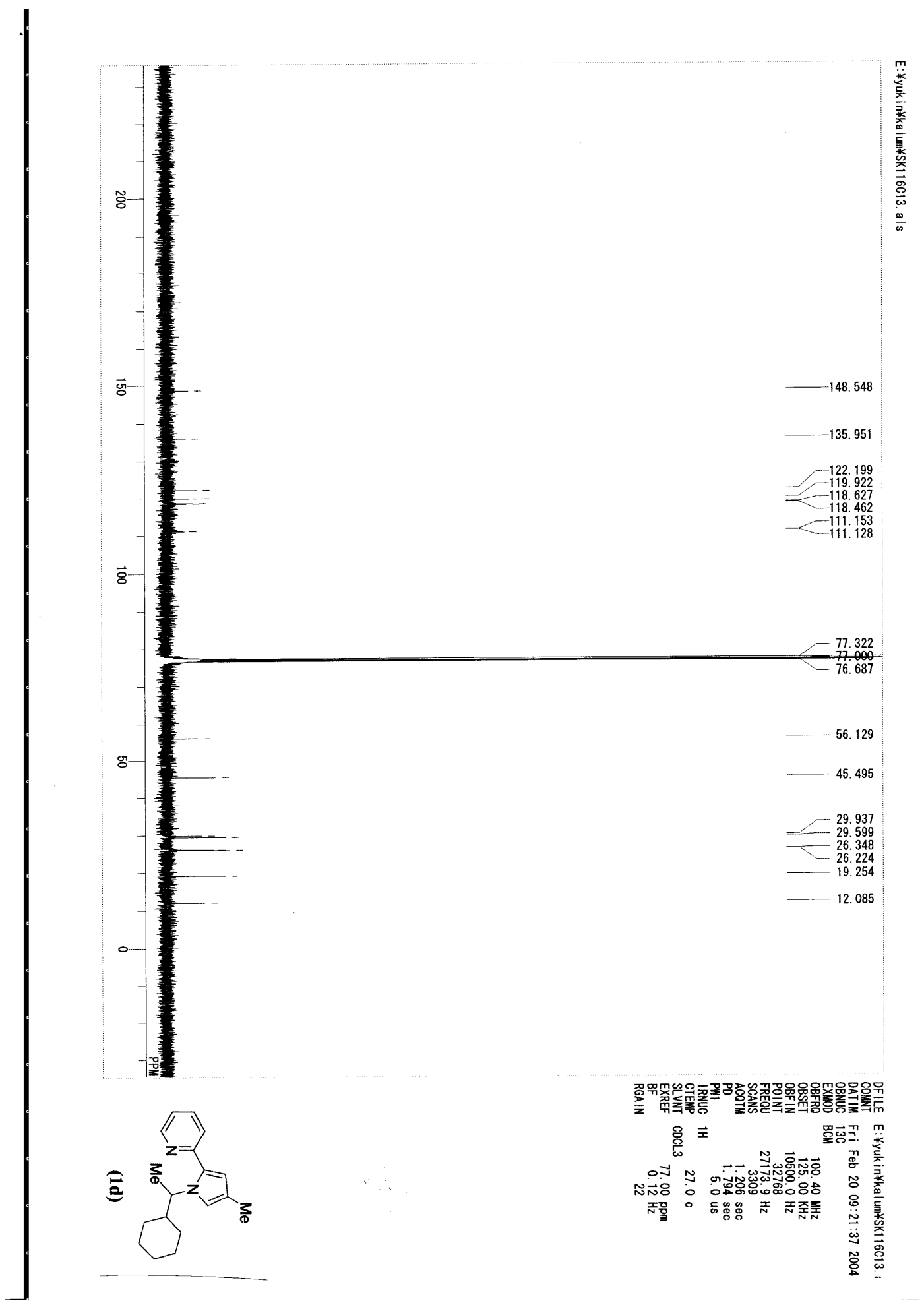


${ }^{1}$ H-NMR of 2-Pyridinyl-\{1-[1-(4-Chloro-phenyl)-ethyl]-4-methyl\}pyrrole (1e).

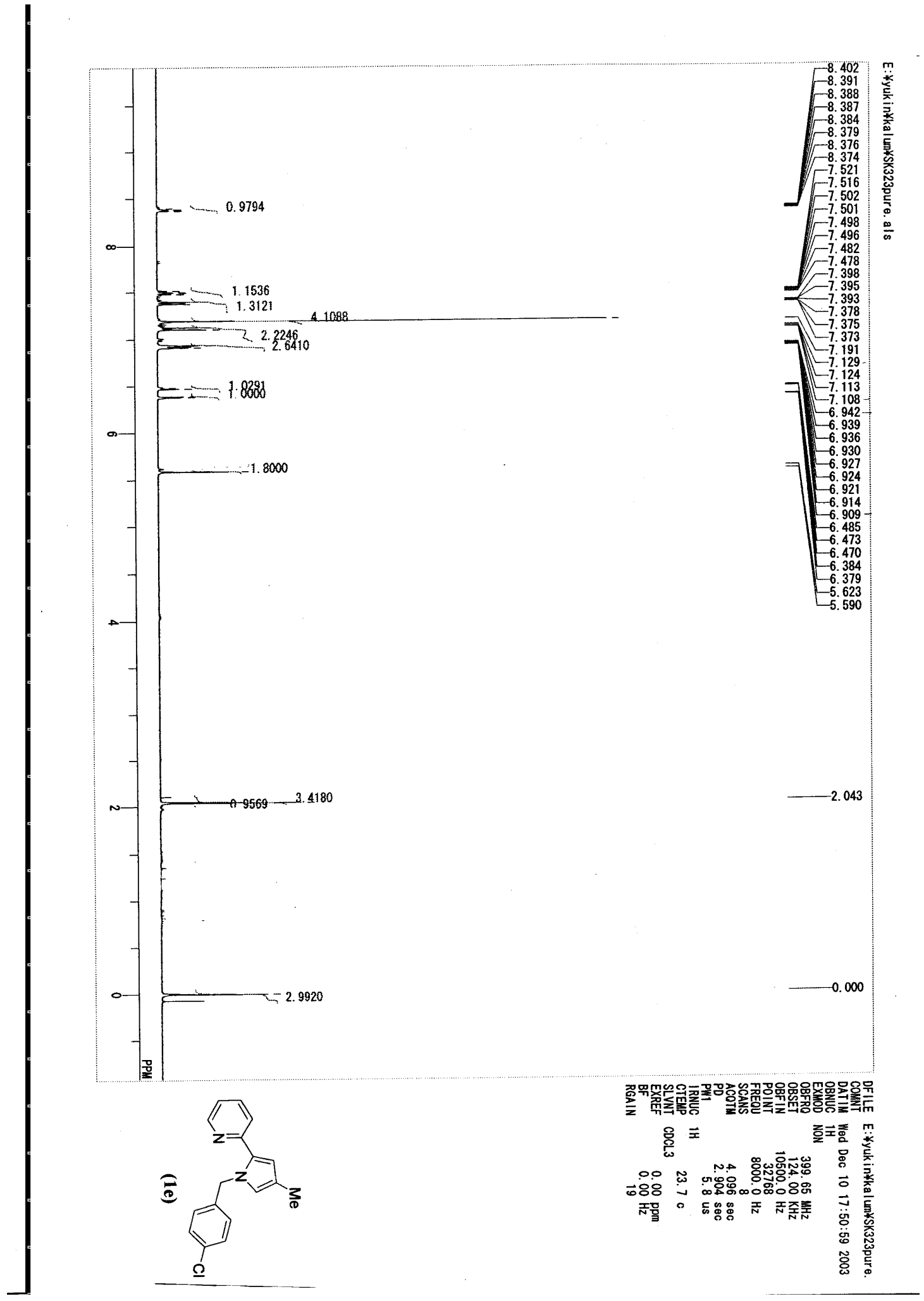


${ }^{13}$ C-NMR of 2-Pyridinyl-\{1-[1-(4-Chloro-phenyl)-ethyl]-4-methyl $\}$ pyrrole (1e).

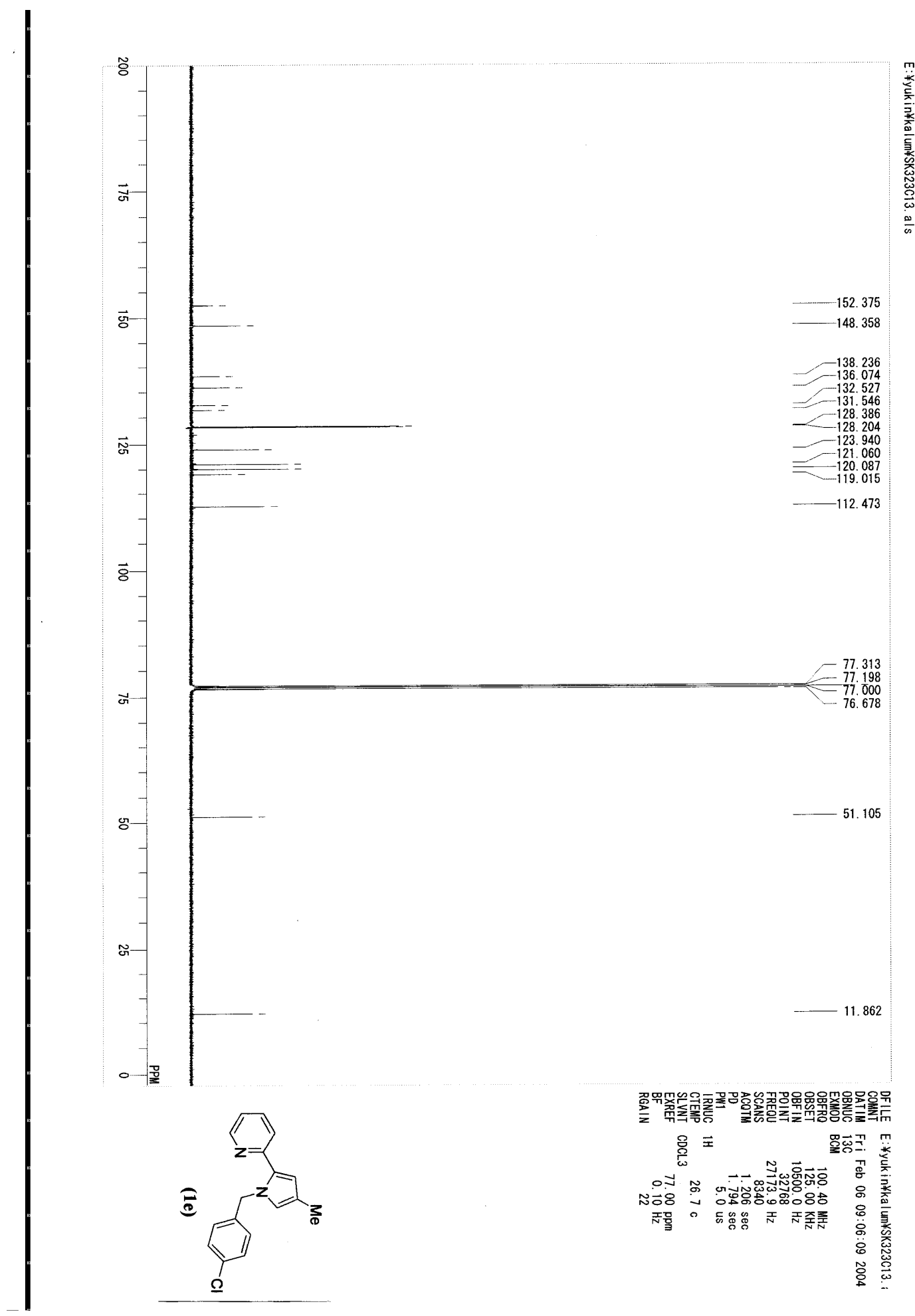


${ }^{1}$ H-NMR of 2-Pyridinyl-[1-(2,2-Dimethoxy-ethyl)-4-methyl]pyrrole (1f).

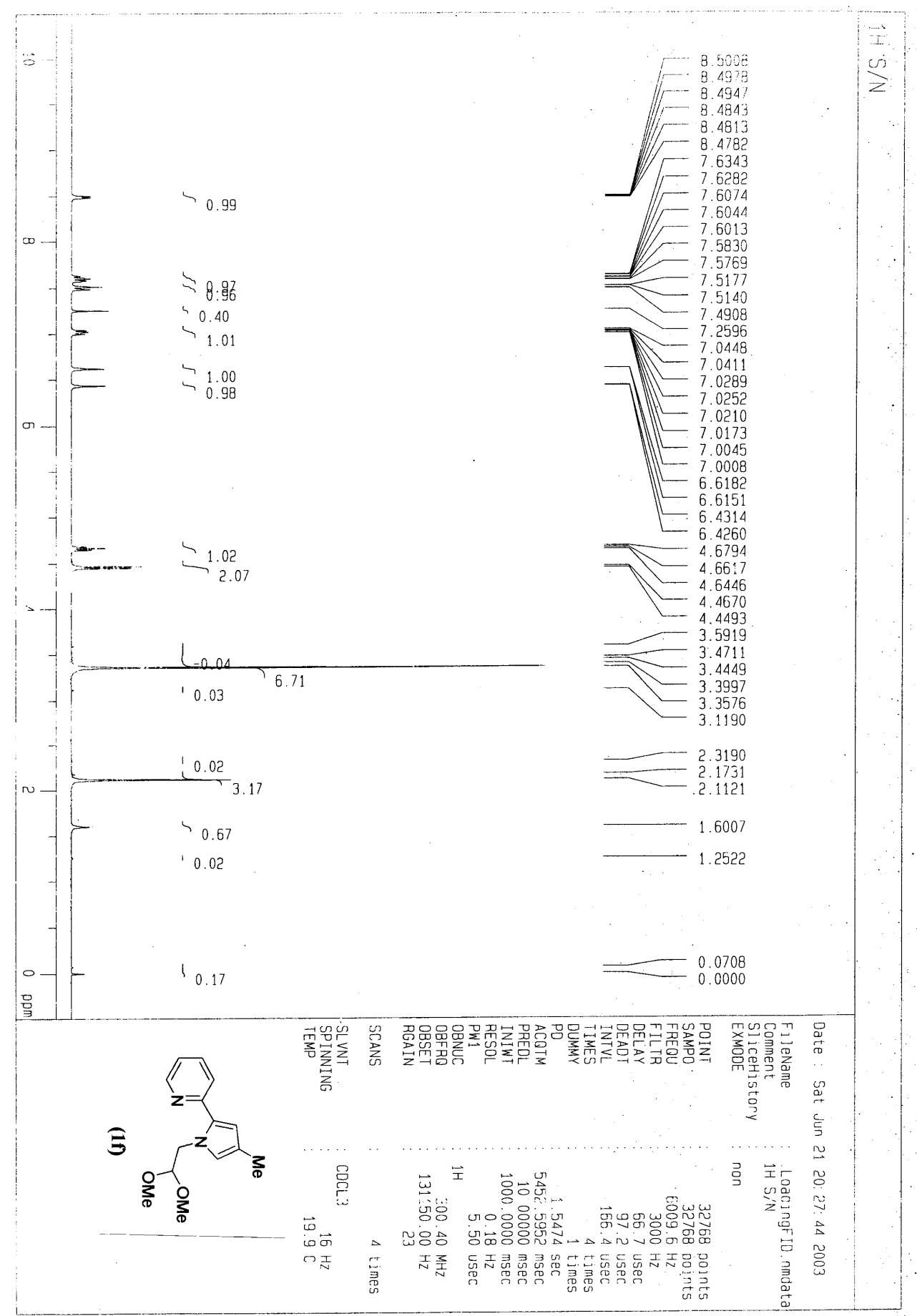


${ }^{13}$ C-NMR of 2-Pyridinyl-[1-(2,2-Dimethoxy-ethyl)-4-methyl]pyrrole (1f).

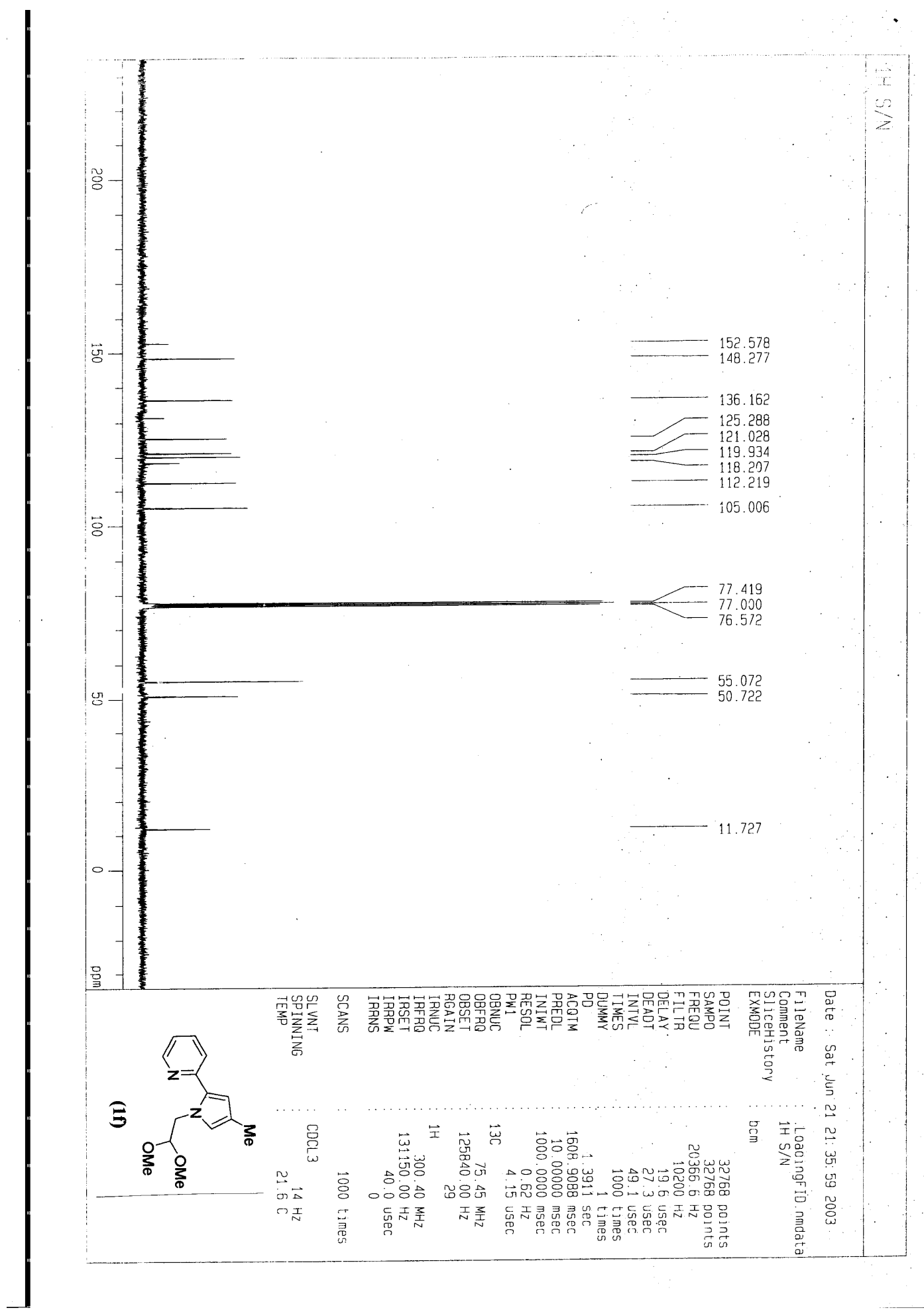


${ }^{1}$ H-NMR of 2-Pyridinyl[1-(3-Methoxy-propyl)-4-methyl]pyrrole (1g).
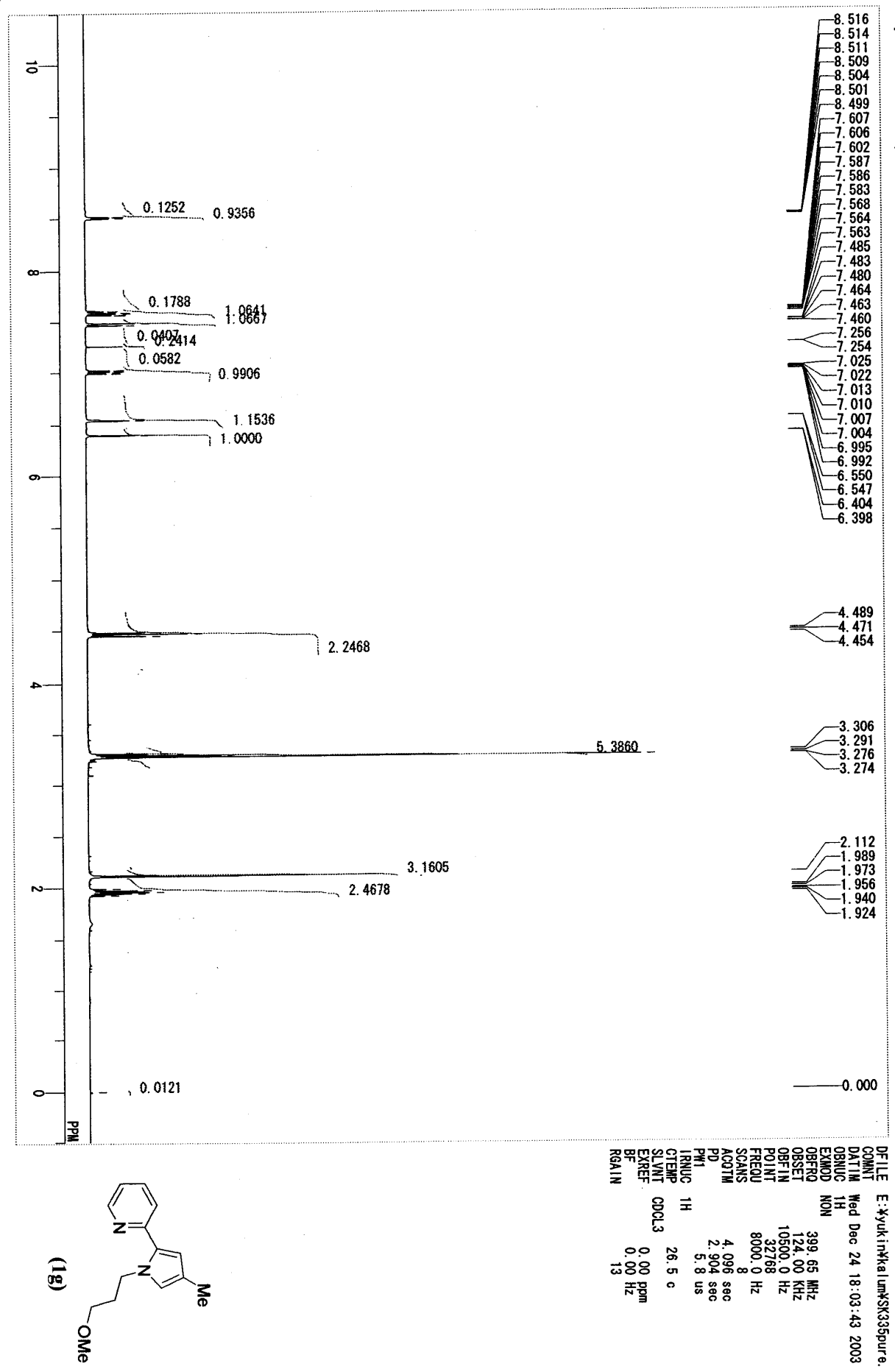
${ }^{13}$ C-NMR of 2-Pyridinyl[1-(3-Methoxy-propyl)-4-methyl]pyrrole (1g).
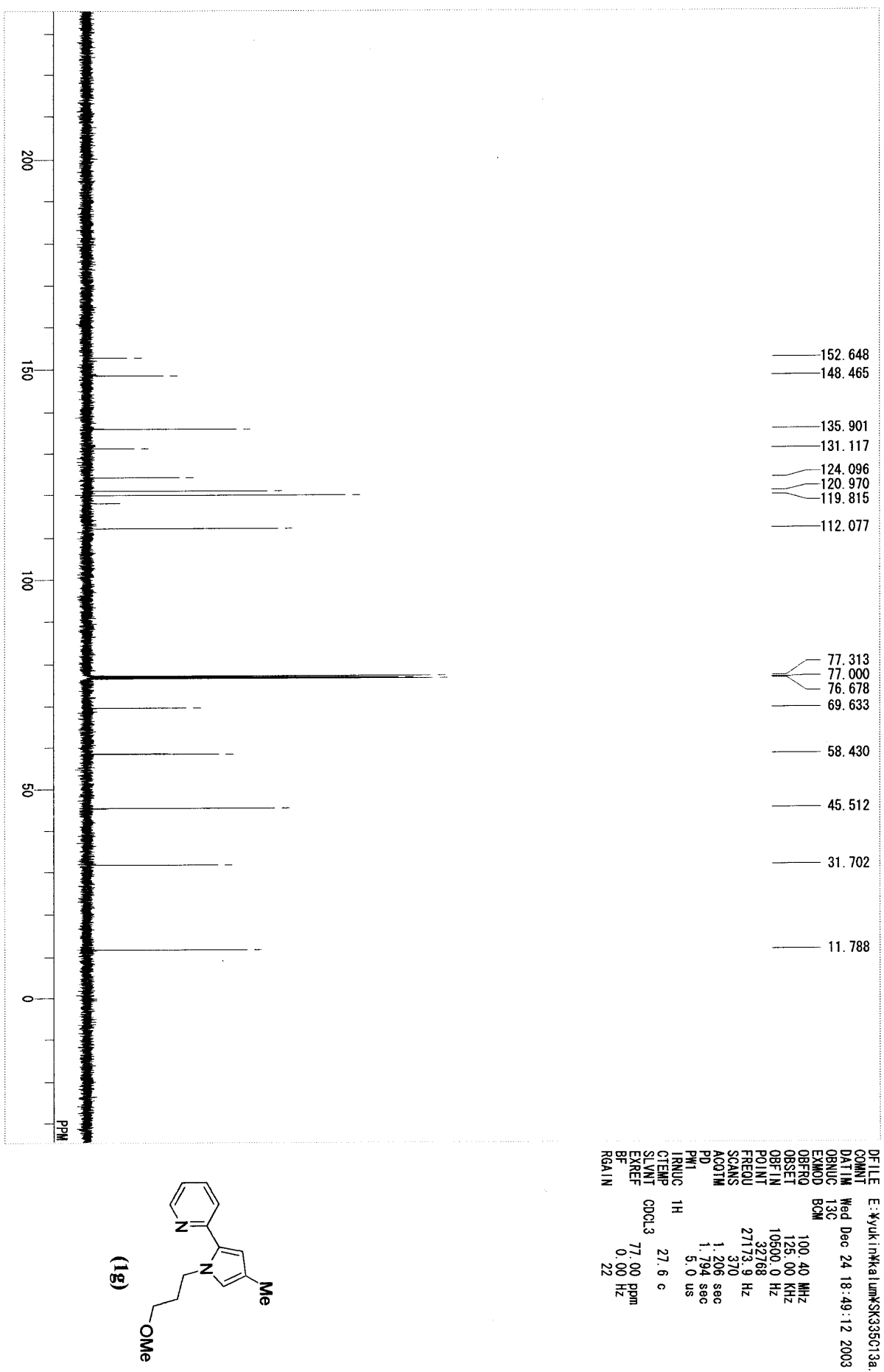
${ }^{1}$ H-NMR of 2-Pyridinyl[4-Methy-1-(1-phenyl-ethyl)]pyrrole (1h).

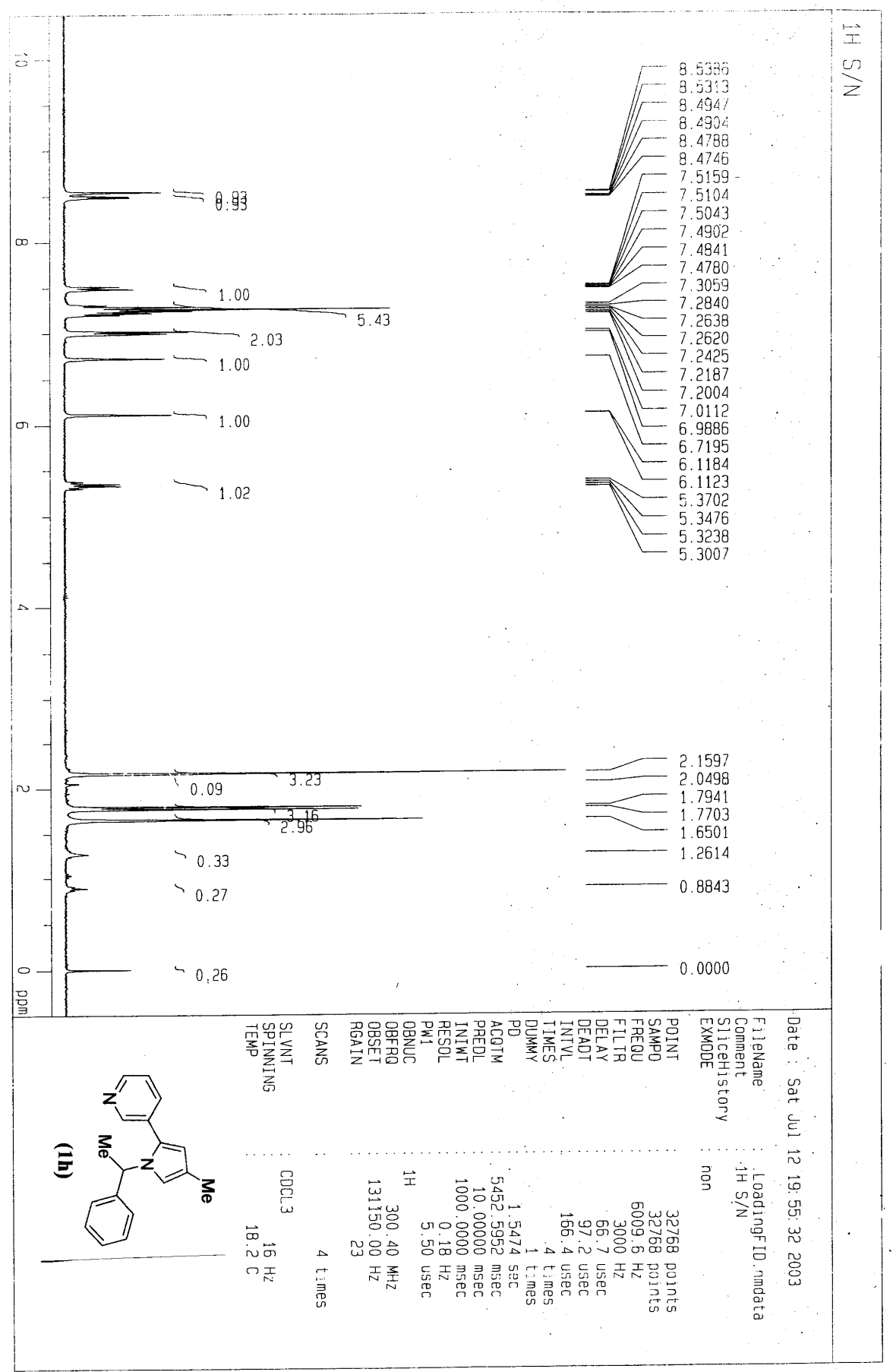


${ }^{13}$ C-NMR of 2-Pyridinyl[4-Methy-1-(1-phenyl-ethyl)]pyrrole (1h).

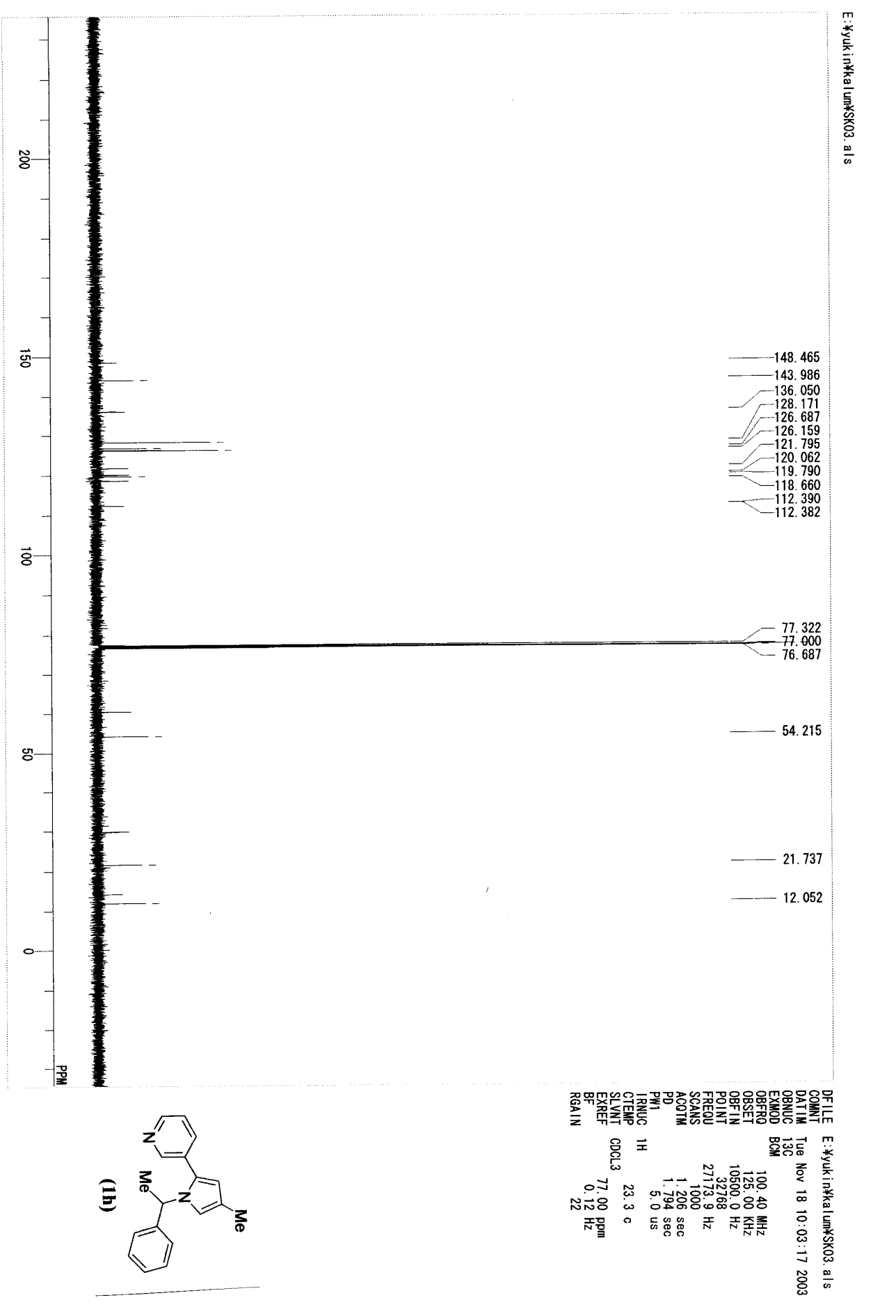


${ }^{1}$ H-NMR of 2-Pyridinyl[Methyl-1-(1-phenyl-ethyl)]pyrrole (1i).

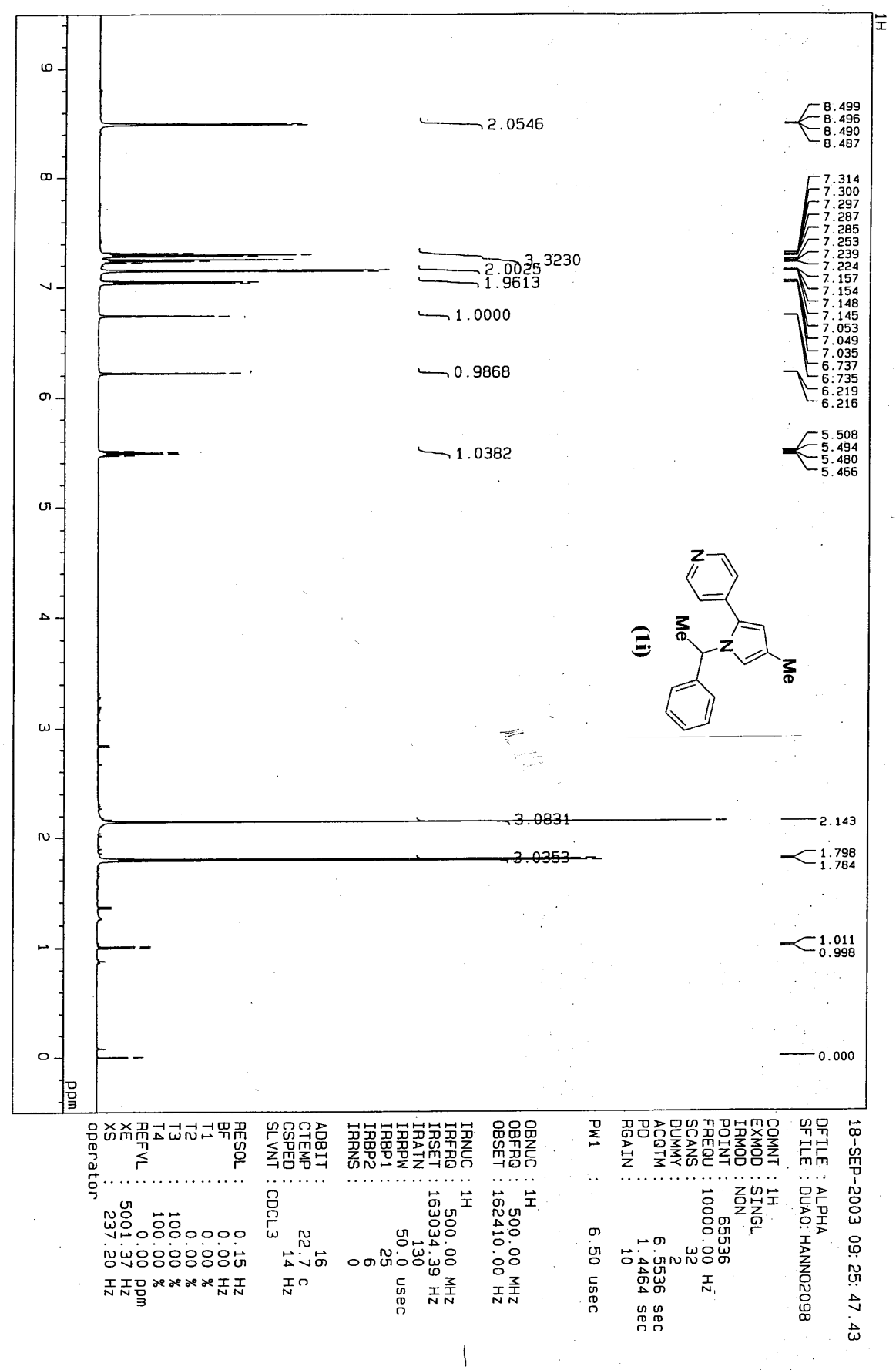




\section{${ }^{13}$ C-NMR of 2-Pyridinyl[Methyl-1-(1-phenyl-ethyl)]pyrrole (1i).}

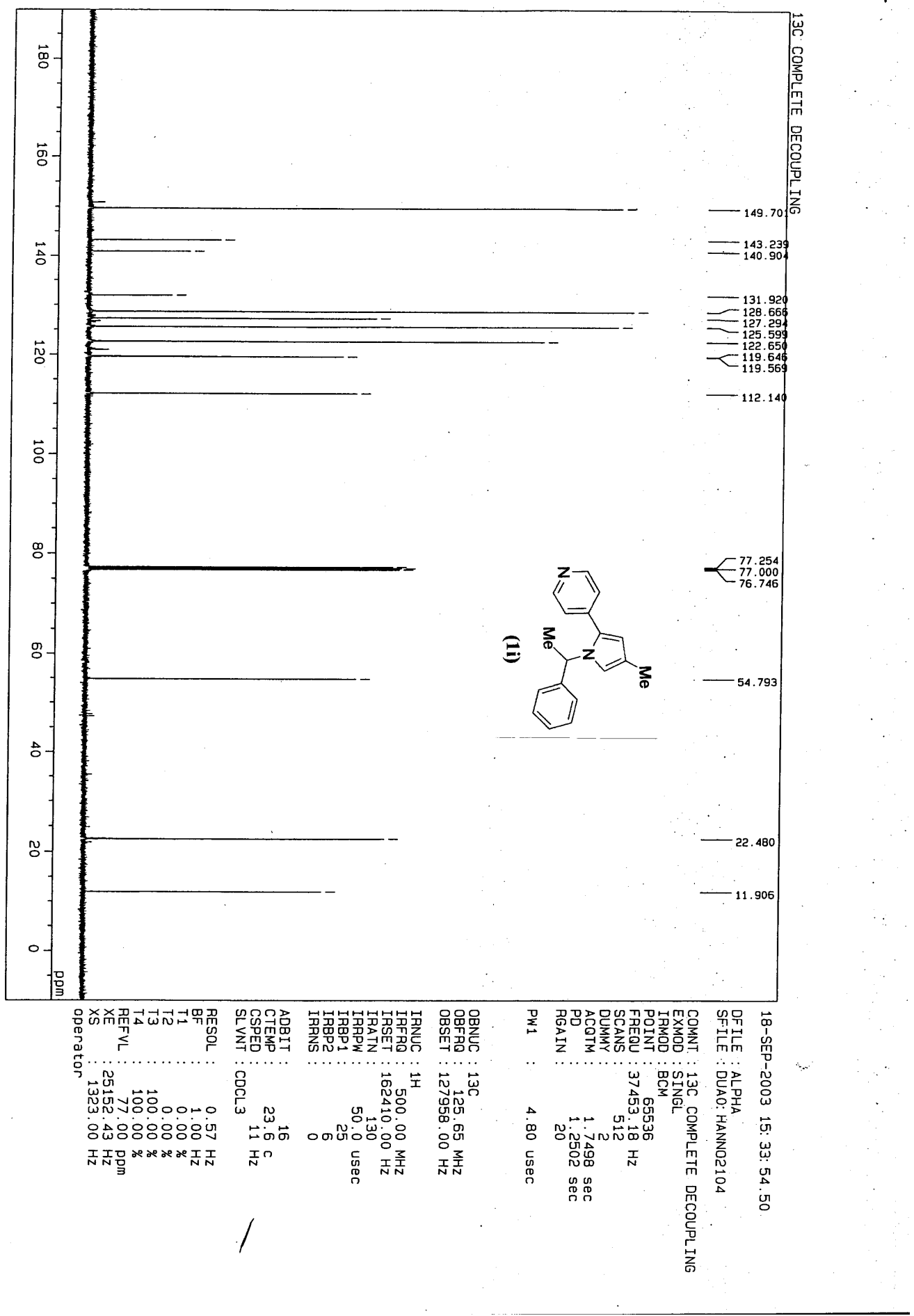


${ }^{1}$ H-NMR of 2-etaneonyl-\{6-[4-Methyl-1-(1-phenyl-ethyl)\}pyrrole (1j).

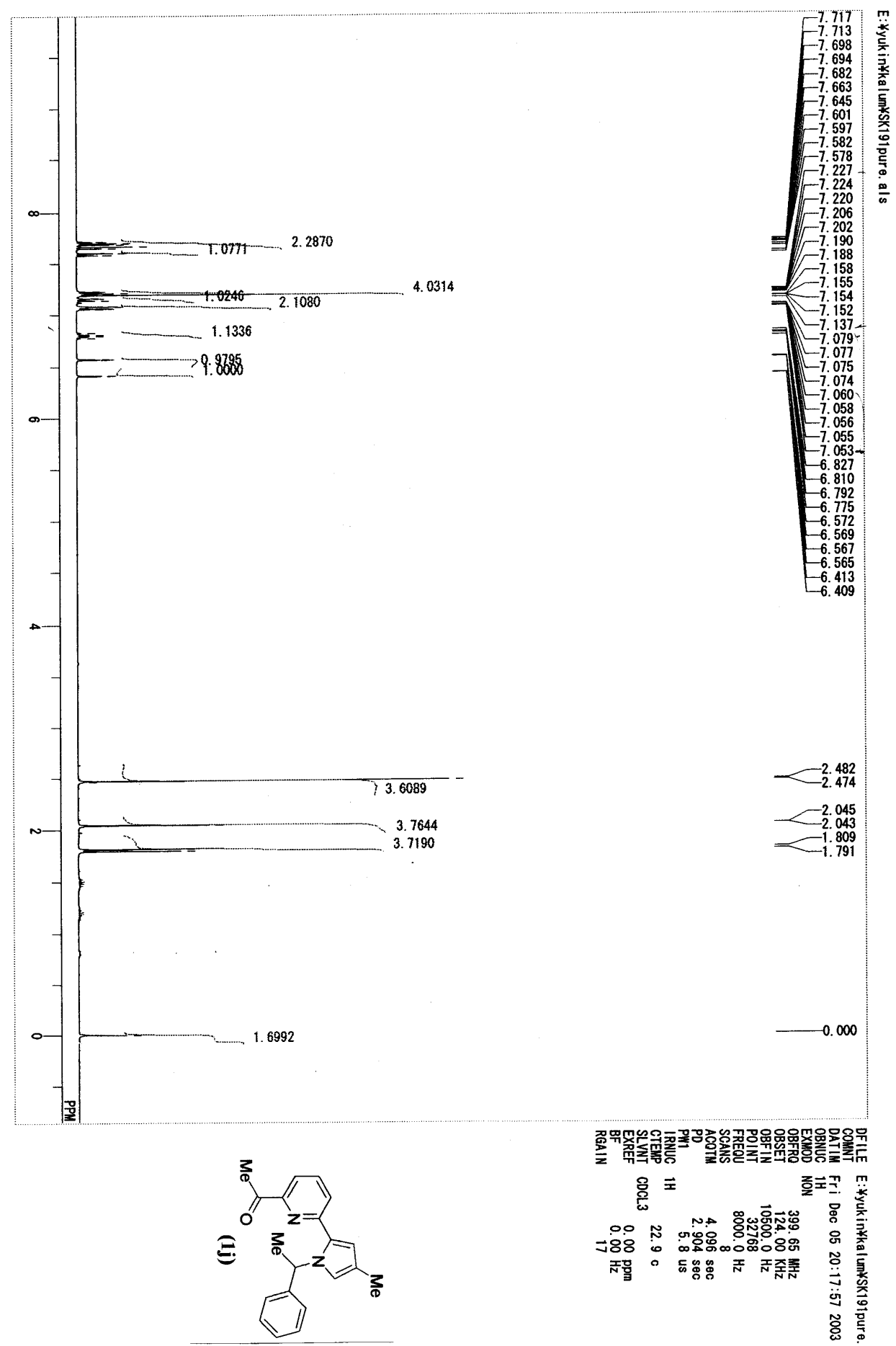


${ }^{13}$ C-NMR of 2-etaneonyl-\{6-[4-Methyl-1-(1-phenyl-ethyl)\}pyrrole (1j).

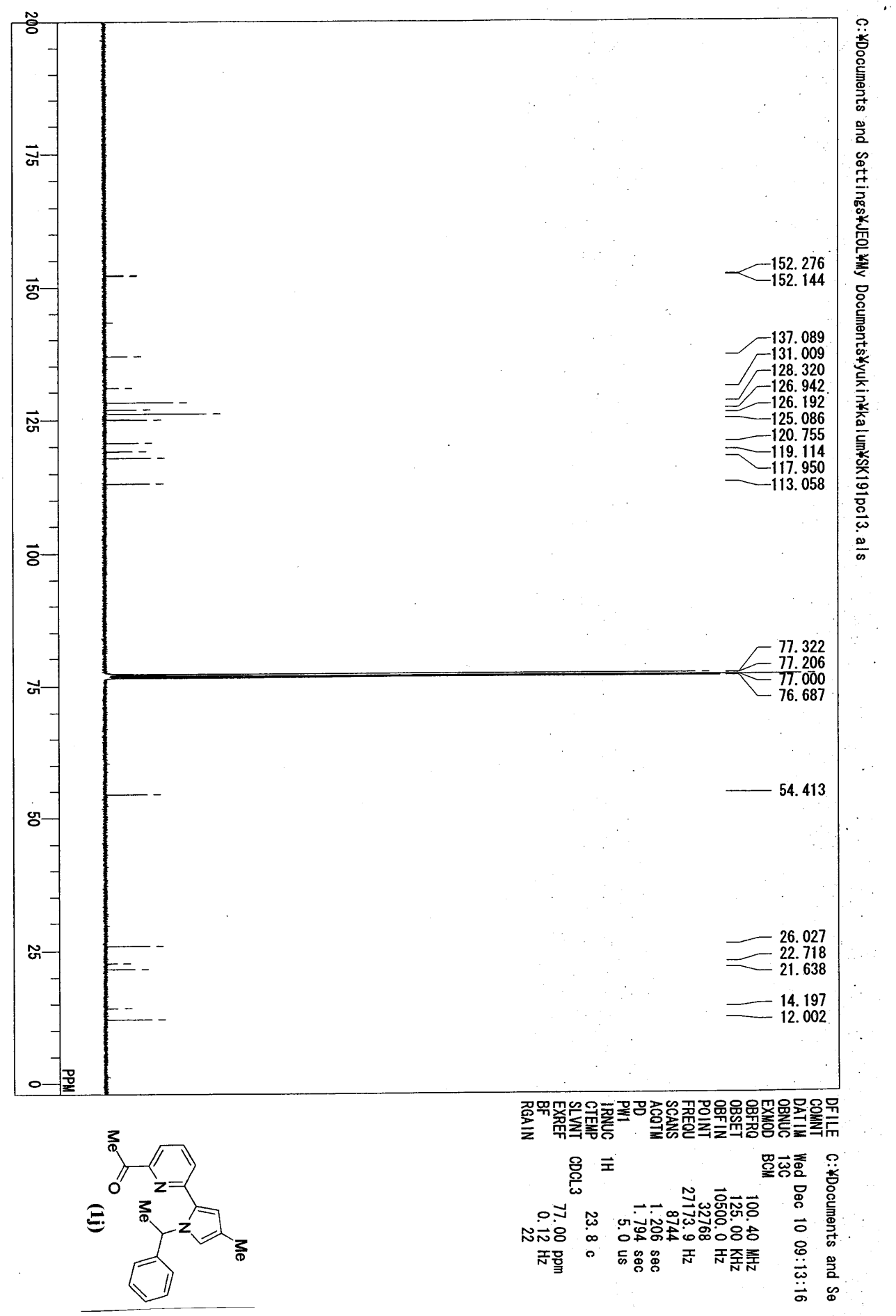




\section{${ }^{1}$ H-NMR of 2-Pyrazinyl-[4-Methyl-1-(1-phenyl-ethyl)]pyrrole (1k).}

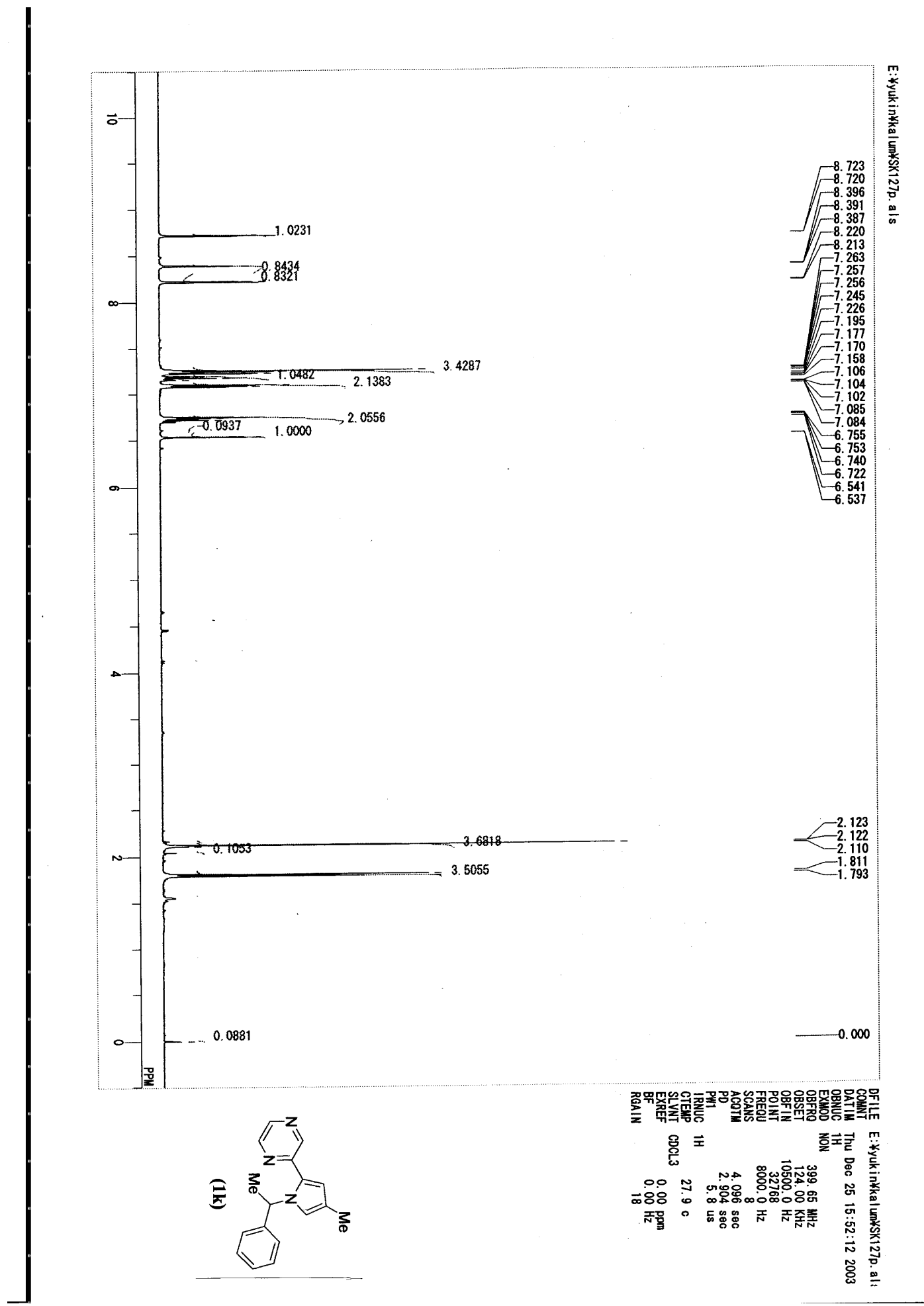


${ }^{13}$ C-NMR of 2-Pyrazinyl-[4-Methyl-1-(1-phenyl-ethyl)]pyrrole (1k).

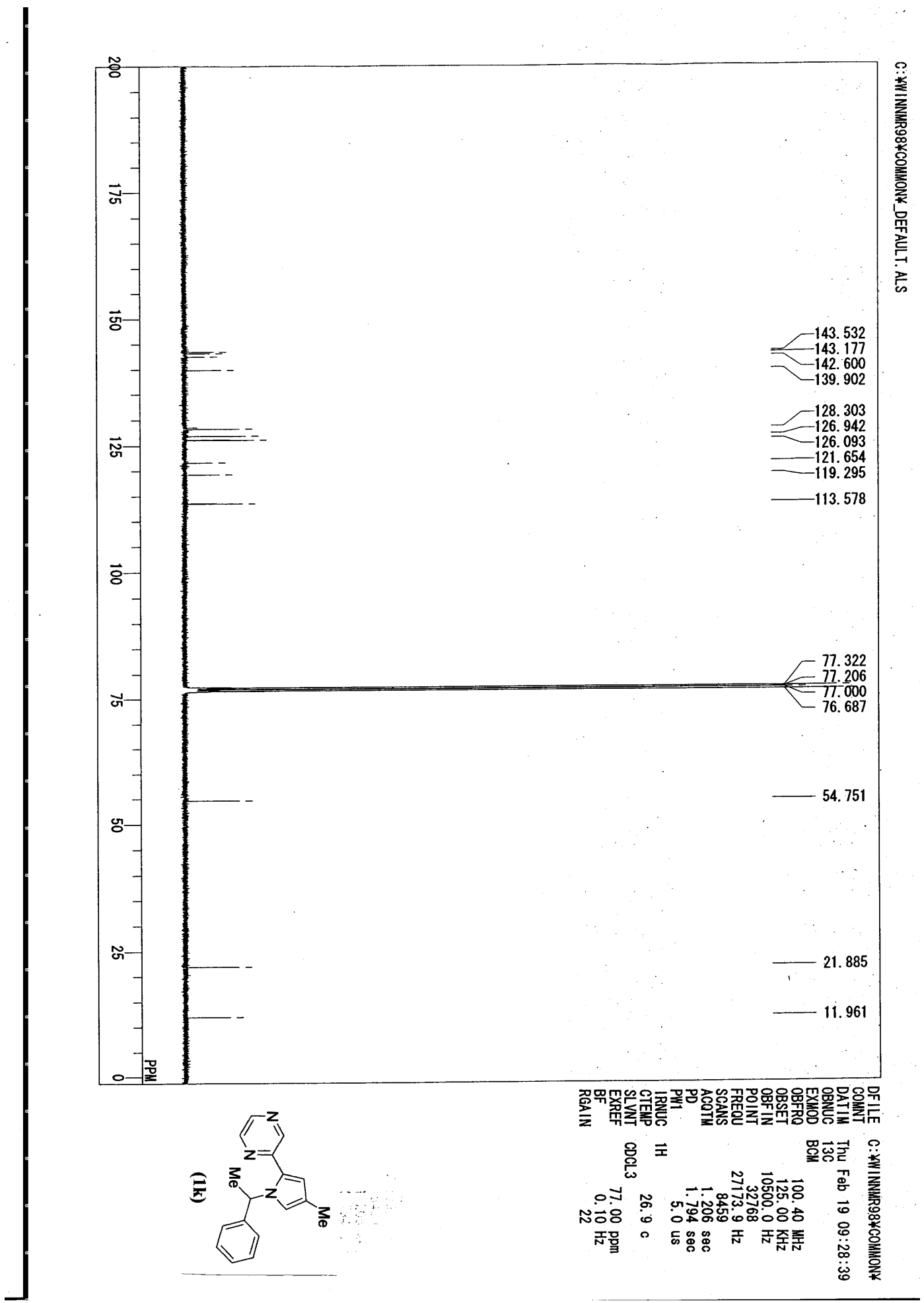




\section{${ }^{1}$ H-NMR of 2-Arylyl-(4-methyl-2-benzyl)pyrrole (10).}

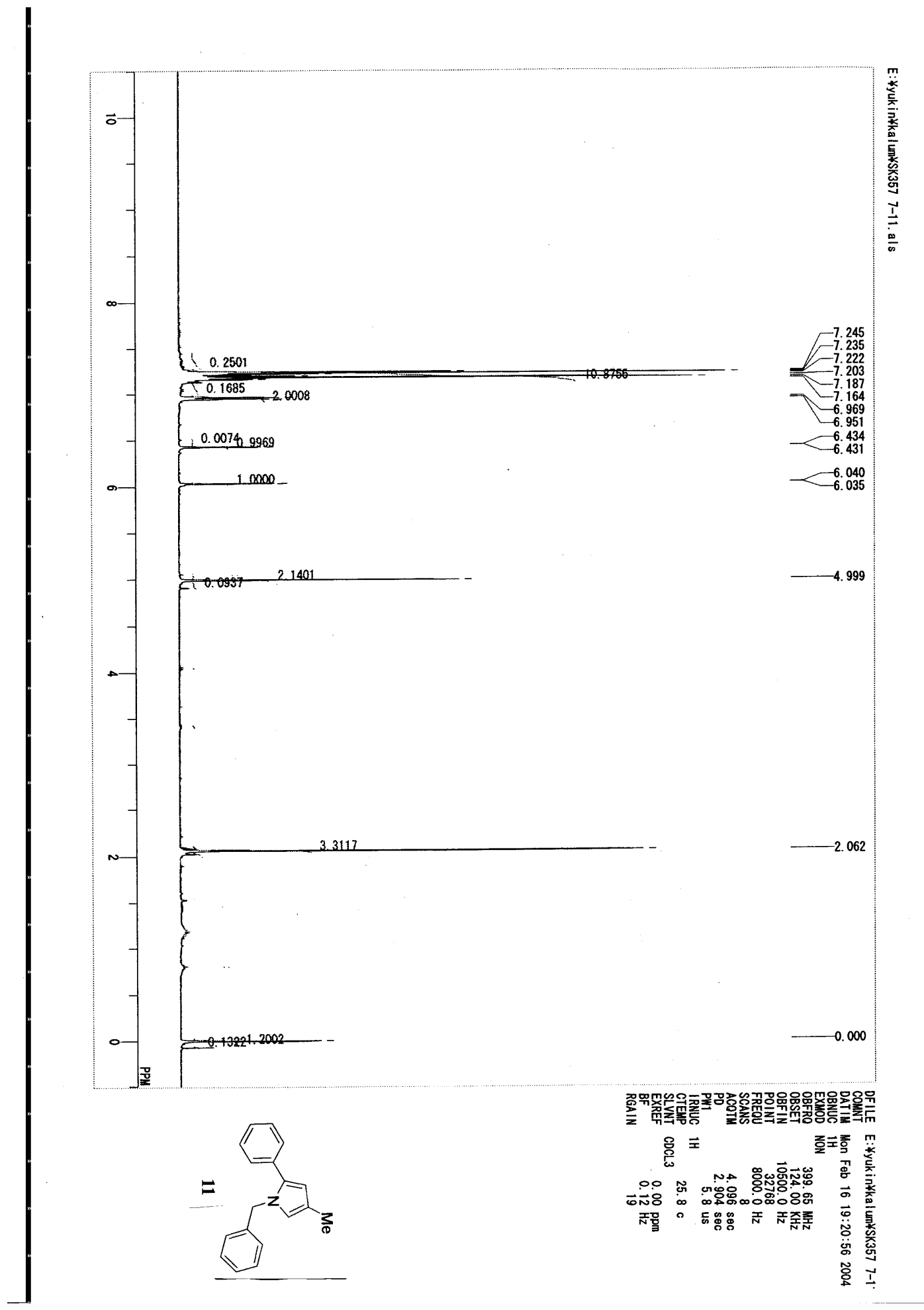


${ }^{13}$ C-NMR of 2-Arylyl-(4-methyl-2-benzyl)pyrrole (10).

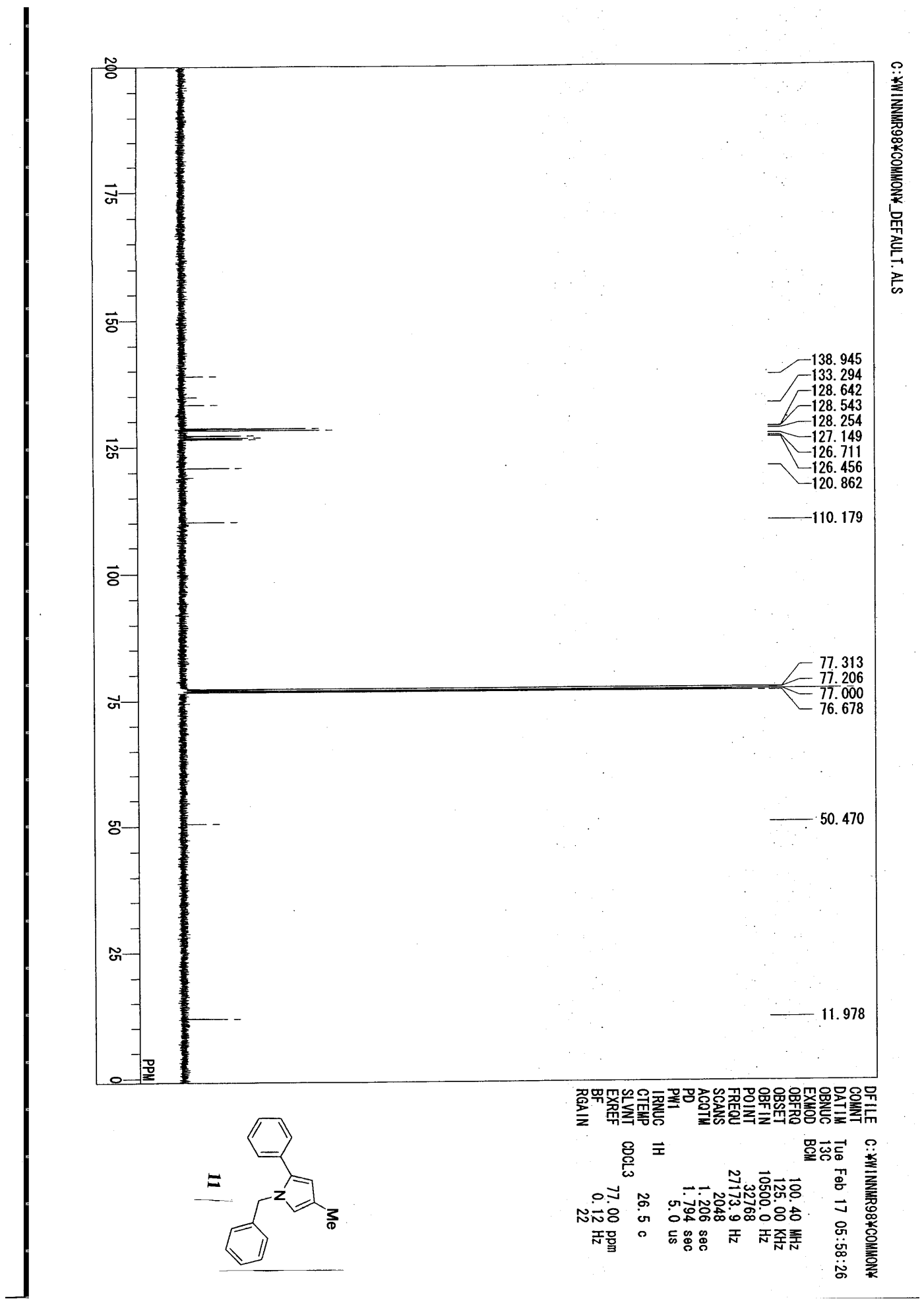




\section{${ }^{1}$ H-NMR of 11.}

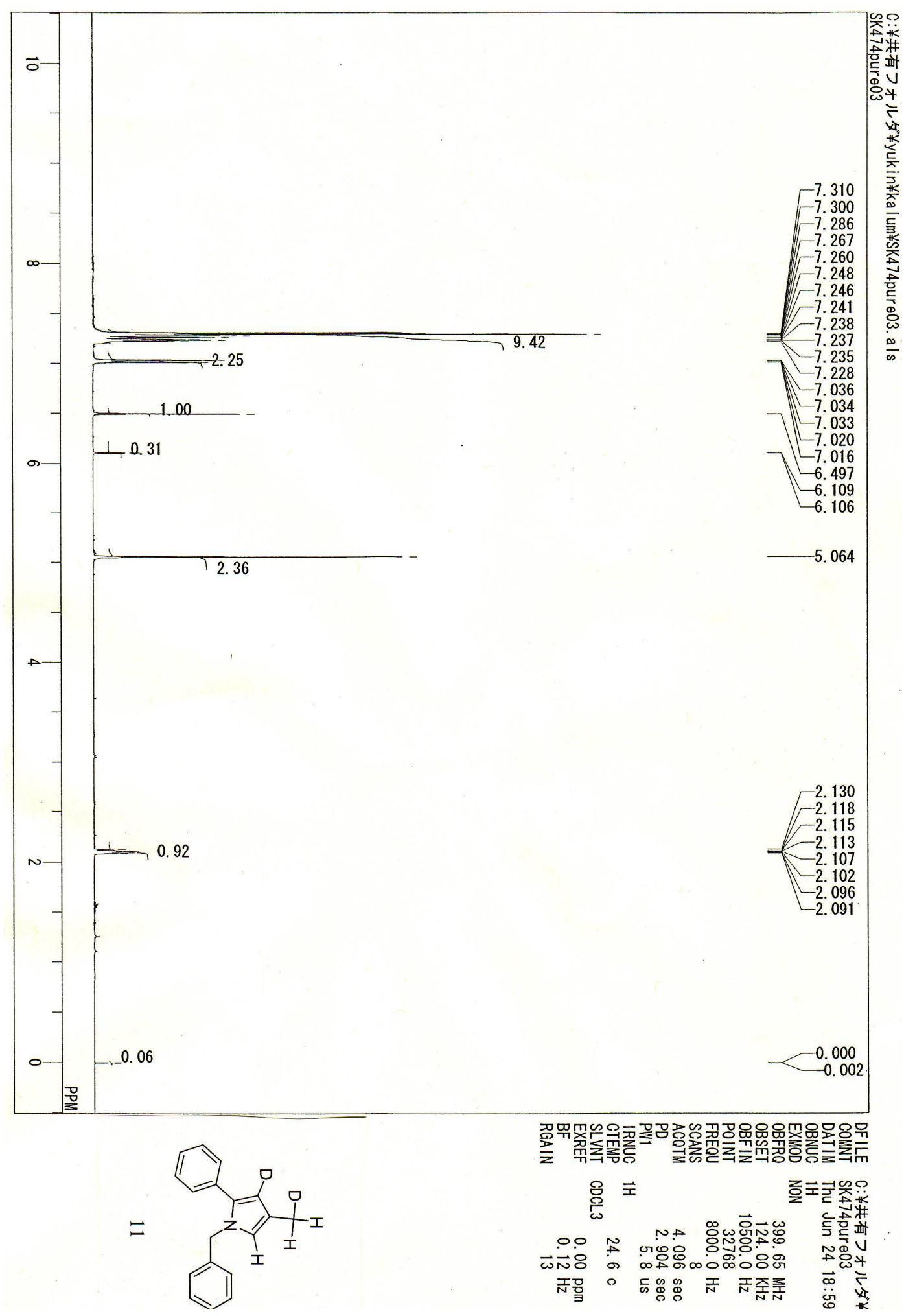


${ }^{1} \mathrm{H}$ NMR of the recovered deuterated acetophenone $\left(9-d_{3}\right)$

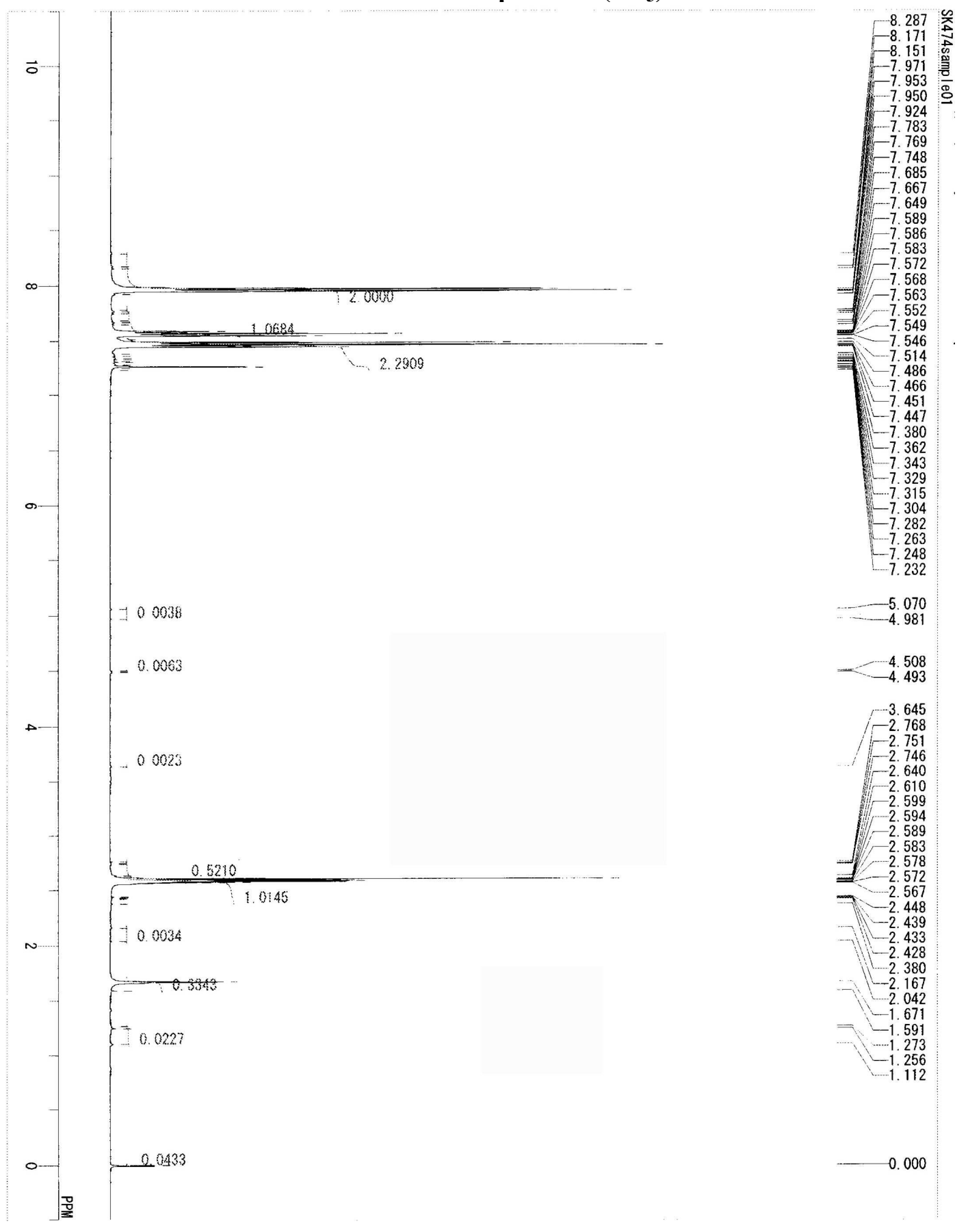

